\title{
Top-down generation of low-precision representations improves the perception and imagination of fine-scale visual information
}

\author{
Zedong $\mathrm{Bi}^{1,2}$ \\ ${ }^{1}$ Institute for Future, Qingdao University, Qingdao 266071, China \\ ${ }^{2}$ School of Automation, Shandong Key Laboratory of Industrial Control Technology, Qingdao University, Qingdao 266071, China \\ Email: zedong.bi@outlook.com
}

\begin{abstract}
Predictive-coding perception or imagination requires top-down generated neural representations in primary visual cortex (V1) to reconstruct or simulate the V1 representations bottom-up stimulated by the seen images. However, top-down generated representations are found to have low coding precision in both perception and imagination tasks. Why and how does the brain use top-down generated low-precision representations to reconstruct or simulate bottom-up stimulated high-precision representations? How can information of fine spatial scale be perceived or imagined using the top-down generated low-precision representations? By modeling visual system using variational autoencoder, we reveal that training to generate low-precision representations facilitates higher-order cortex to form representations smooth to shape morphing of stimuli. Fine-scale visual information, if sparse, can be more accurately and robustly perceived or creatively imagined by inferring from generated representations with low (instead of high) coding precision. Therefore, generating low-precision representations is not a shortcoming, but instead a strategy that the brain uses to improve the perception and imagination of fine-scale information.
\end{abstract}

\section{Introduction}

Analysis-by-synthesis is a long-standing perspective of perception, which proposes that instead of passively responding to external stimuli, the brain actively predicts and explains its sensations $[1,2]$. Neural network models in this line use a generative network to reconstruct the external stimuli, and use this reconstruction error to guide the updating of the neuronal dynamic states [3] or synaptic weights [4, 5]. This analysis-by-synthesis perspective of perception has received experimental supports in both visual and auditory systems [2, 6]. Additionally, neural representations are generated in a top-down cascade during visual creative imagination [7], and these representations are highly similar to those activated during perception $[8,9]$. These results above suggest that the brain performs like a top-down generator in both perception and imagination tasks.

Top-down generation is believed to be an essential basis of consciousness [10,11]. Feedback transmission needs to be ignited for the awareness of visual stimuli $[12,13,14]$. High-level mental operations such as perceptual integration, working memory and executive control, can happen unconsciously [15,16,17], if stimuli are transmitted feedforwardly without triggering the feedback pathway. Global workspace theory proposes that consciousness happens when a mental model of sensory information is broadcasted to the whole brain by topdown connections, with the broadcasted mental model updated according to the mismatch between model-predicted and observed data [14]. Moreover, imagination, the generation of fictional representation using the internal model of the real world through top-down connections [18], plays an important role in prospective planning and retrospective analysis [10, 19, 20], and is key in model-based reinforcement learning [21]. 
bioRxiv preprint doi: https://doi.org/10.1101/2021.05.07.443208; this version posted August 30, 2021. The copyright holder for this preprint

(which was not certified by peer review) is the author/funder, who has granted bioRxiv a license to display the preprint in perpetuity. It is made available under aCC-BY-NC-ND 4.0 International license.

However, in both perception and imagination, top-down generated representations have lower coding precision than the representations bottom-up stimulated by seen images. It has been found that V1 neurons have larger receptive fields (RFs) and are tuned to lower spatial frequencies in imagined images than in seen images [22, 23]. If one agrees that imagination requires top-down generated representations to simulate the representations stimulated by seen images $[8,18,9]$, one should be curious about why and how the brain uses the generated lowprecision representations to imagine, especially the visual information with fine spatial scale which is believed to be coded by $\mathrm{V} 1$ neurons that have small RFs and prefer to high spatial frequencies. During perception, the responses of V1 neurons to stimuli in classical RFs are modulated by extra-classical RFs that surround classical RFs [24]. This surround modulation, which is mediated by feedback connections [25], is believed to reflect the reconstruction of V1 activity by higher-order cortex in predictive-coding perception [3]. However, extraclassical RFs also have lower precision than classical RFs. Extra-classical RFs have larger size (i.e., lower spatial precision) than classical RFs. The modulation of classical RFs by extra-classical RFs is insensitive to grating phase even for simple cells, and has broader tuning to spatial frequencies than classical RFs [26]. If one agrees the predictive-coding theory of perception [3], one should be curious about why and how the brain uses the generated low-precision representations in extra-classical RFs to reconstruct the stimulated high-precision representations in classical RFs, especially for the visual information with fine spatial scale coded in the small-sized classical RFs of the phase-sensitive simple cells. Overall, the computation underlying the mismatch between the coding precision of the top-down generated and bottom-up stimulated representations remains unclear. Understanding this computation is important due to the fundamental role of perception and imagination in cognition (discussed in the previous paragraph).

In this paper, we address these problems by modeling the visual system as a variational auto-encoder (VAE) [27]. We show that training to generate low-precision representations facilitates higher-order cortex to form representations smooth to shape morphing of stimuli, thereby improving visual perceptive accuracy and robustness as well as imaginative creativity. Fine-scale information can be faithfully inferred from low-precision representations if fine-scale information is sparse. Our results suggest that generating low-precision representations that contain sparse fine-scale information is an important strategy the brain uses to improve perception and imagination of fine-scale information, and reveal the computational advantage of sparse code in V1 from top-down generative perspective.

\section{Results}

\section{Low-precision representations can be better simulated or reconstructed through top-down pathway}

\section{Model setup}

We model the visual system as a variational auto-encoder (VAE) [27], whose output is optimized to reconstruct the input (Figure 1a). In response to any input, the bottleneck state is sampled from a Gaussian distribution whose mean and variance are determined by the output of the encoder part (Figure 1a). This Gaussian distribution is encouraged to be close to standard normal distribution during training. VAE mimics the visual system in the following four aspects: (1) the bottleneck layer $\mathcal{B}$, which is low-dimensional, can be regarded as the prefrontal cortex, because experiments suggest that working memory is maintained in a low-dimensional subspace of the state of the prefrontal cortex [28, 29]; (2) the input $\mathcal{I}$ and output $\mathcal{O}$ layers can be regarded as V1, with the $\mathcal{I} \rightarrow \mathcal{B}$ and $\mathcal{B} \rightarrow \mathcal{O}$ connections (i.e., the encoder and decoder parts) representing the bottom-up and top-down pathways respectively; (3) random sampling is a designed ingredient of VAE, analogous to neuronal stochasticity; and (4) after training, VAE can generate new outputs through the top-down pathway by sampling states of the bottleneck layer from standard normal distribution, mimicking the top-down generation in mental imagination [8, 18], and perception can also be modeled through an error-correcting process (see below). This fourth point makes VAE be a generative model of the visual system.

Modeling the visual system using VAE has also been suggested by other authors [10]. The fourth point above suggests that VAE provides a unified model for imagination and perception. The cost function that VAE minimizes is the variational free energy [27]. Minimizing variational free energy is a unified theoretical framework to understand perception and learning [1]. Modeling the visual system using VAE expands the explanation power of this framework to imagination.

We considered three toy models of V1 activity, in each of which V1 neurons are located on a 1-dimensional line. In Model 1, only a single neuron has non-zero activity, while all the other neurons are silent (Figure 1b, left). In Model 2, the profile of neuronal activities is an 

available under aCC-BY-NC-ND 4.0 International license.

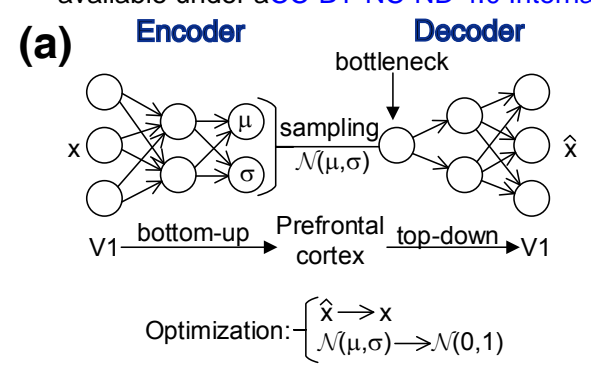

(b)
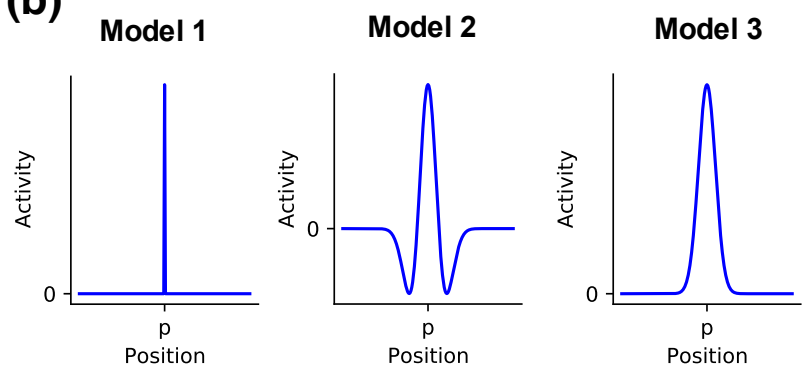

Figure 1: The models to study the generation of representations on a 1-dimensional line. (a) Schematic of the variational auto-encoder (VAE). The value $z$ of the bottleneck (which models the prefrontal cortex) is sampled from a Gaussian distribution $\mathcal{N}(\mu, \sigma)$, with $\mu$ and $\sigma$ determined by the two output channels of the bottom-up pathway. This network is optimized so that the output of the top-down pathway $\hat{x}$ is close to the input stimulus $x$, and at the same time $\mathcal{N}(\mu, \sigma)$ is close to $\mathcal{N}(0,1)$. (b) Profiles of the three models of $\mathrm{V} 1$ activity. The peak (Model 1), wavelet (Model 2) and bump (Model 3) are parameterized by their positions $p$ on a 1-dimensional line.

oscillating wavelet (Figure 1b, middle). In Model 3, the profile of neuronal activities is a Gaussian bump (Figure 1b, right). The shapes of the three activity profiles are parameterized by their positions on a one-dimensional line. We trained VAE to generate the activity patterns of the three models, and compared the quality of the generated patterns in imagination and perception tasks.

These three models can be biologically interpreted as follows:

(1) The neuronal activities in the three models can be regarded as the responses of three types of V1 simple cells to a dot stimulus on a 1-dimensional line. The sizes of the receptive fields of the simple cells in Model 1 are close to zero, smaller than those in Model 3. By comparing the patterns generated by VAEs trained by the activity patterns in Model 1 and Model 3, we can study the influence of receptivefield size to imagination and perception. Additionally, the receptive fields in Model 2 have the same spatial scale of Gaussian decay with the receptive fields in Model 3, but have higher spatial frequency. So we can study the influence of spatial-frequency preference to imagination and perception by comparing the images generated by VAEs trained by Model 2 and Model 3. Experiments have found that V1 neurons have larger receptive fields and are tuned to lower spatial frequencies in imagined images than in seen images [22, 23]. Comparing Model 3 with Models 1 and 2 brings understanding on this phenomenon.

(2) We can also interpret stimuli in the spatial-frequency domain. Neurons in Model 1 have sharp spatial-frequency tuning, whereas neurons in Model 3 have broad spatial-frequency tuning. Experiments have found that the top-down modulated extra-classical receptive fields have broader spatial-frequency tuning than the bottom-up classical receptive fields [26]. Comparing these two models brings understanding on this phenomenon.

(3) Neurons in Model 3 can be regarded as the complex cells corresponding to the simple cells in Model 1 and Model 2. So by comparing Model 1 or Model 2 with Model 3, we can also study the influence of phase sensitivity to imagination and perception. Experiments have found that extra-classical receptive fields are insensitive to grating phase even for simple cells [26], which can be understood by comparing Model 3 with Models 1 and 2. 


\section{Visual imagination} available under aCC-BY-NC-ND 4.0 International license.

Visual imagination is modeled by sampling the bottleneck state $z$ of a trained VAE from standard normal distribution, so that the decoder part of VAE (Figure 1a) outputs various activity patterns. To get a detailed understanding on the relationship between $z$ and the generated pattern, we let $z$ to be 1-dimensional, and investigated how the generated pattern changed with $z$. In Model 1, sharp peaks were never generated by VAE, suggesting the failure of pattern generation (Figure 2a). In Model 2, abrupt changes of wavelet positions sometimes happened with the continuous change of $z$ (Figure 2b). In Model 3, Gaussian bumps were generated, with the bump position smoothly changing with $z$ (Figure 2c). These results suggest that generating low-precision representations in V1 (i.e., the representations resulting from large receptive fields, low spatial-frequency preference and phase invariance) facilitates higher-order cortex to form representations smooth to shape morphing (which is here the translational movement of the activity profiles along the 1-dimensional line) of stimuli.

(a)
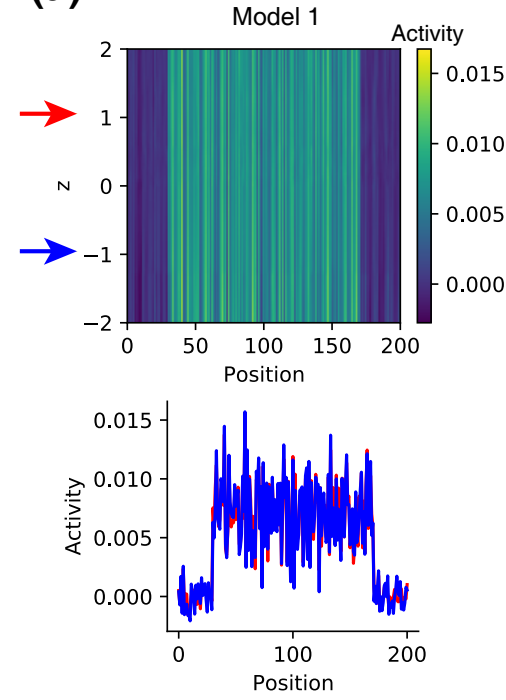

(d)

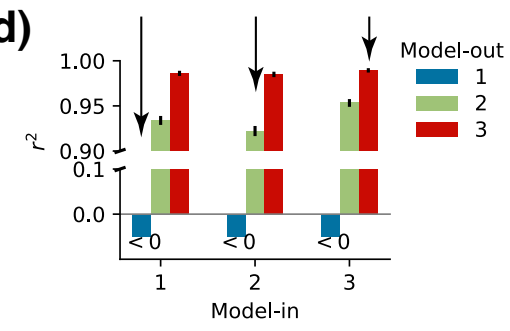

(b)
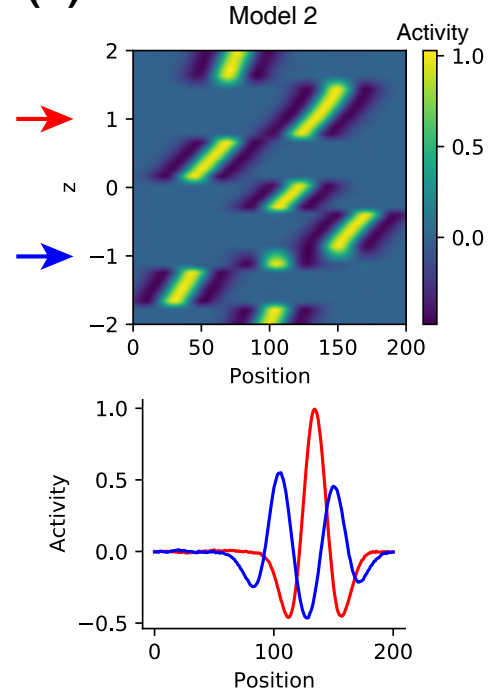

(e) (c)
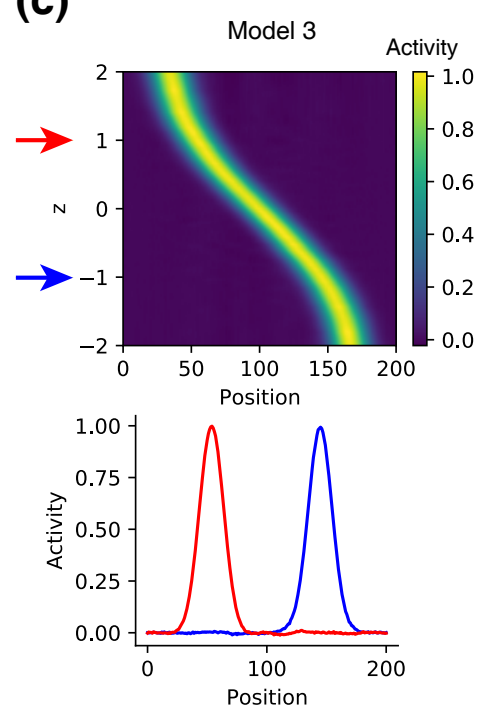

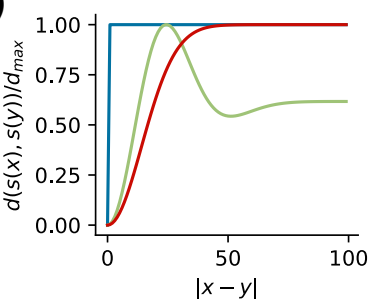

Figure 2: VAE is better at generating low-precision representations. (a) Upper panel: generated activity patterns as a function of the state $z$ of the bottleneck variable. Lower panel: blue and red curves respectively represent the two generated patterns when $z$ takes the two values ( -1 and 1$)$ indicated by the red and blue arrows in the upper panel. VAE is trained using the activity pattern of Model 1. (b, c) Similar to panel a, except that VAE is trained using the activity pattern of Model 2 (panel b) or 3 (panel c). (d) We let the input of VAE (i.e., $x$ in Figure 1a) be the activity pattern of Model-in, but train the output of VAE (i.e., $\hat{x}$ in Figure 1a) to approach the activity pattern of Model-out. Both Model-in and Model-out can be Model 1, 2 or 3. We do not accurately show the value of $r^{2}$ when $r^{2}<0$. Arrows indicate the cases when Model-in equals Model-out, which are the cases in panels a-c. Error bars represent standard error of mean (s.e.m.) over 1200 samples. (e) Euclidean distance $d(s(x), s(y))$ between two activity patterns $s$ as a function of the distance $|x-y|$ between the positions $x$ and $y$ of two dot stimuli on the 1-dimensional line, normalized by the maximal value of $d$, for Models 1 (blue), 2 (green) and 3 (red).

To quantify the quality of the generated patterns, we defined an index $r^{2}$ to indicate how well a VAE-generated pattern looks like an activity pattern of the model used to train the VAE (see Methods). We found $r^{2}$ is maximal for Model 3, intermediate for Model 2, but is small (even negative) for Model 1 (see the bars indicated by arrows in Figure 2d). This result suggests that low-precision representations can be better simulated through top-down pathway. 

available under aCC-BY-NC-ND 4.0 International license.
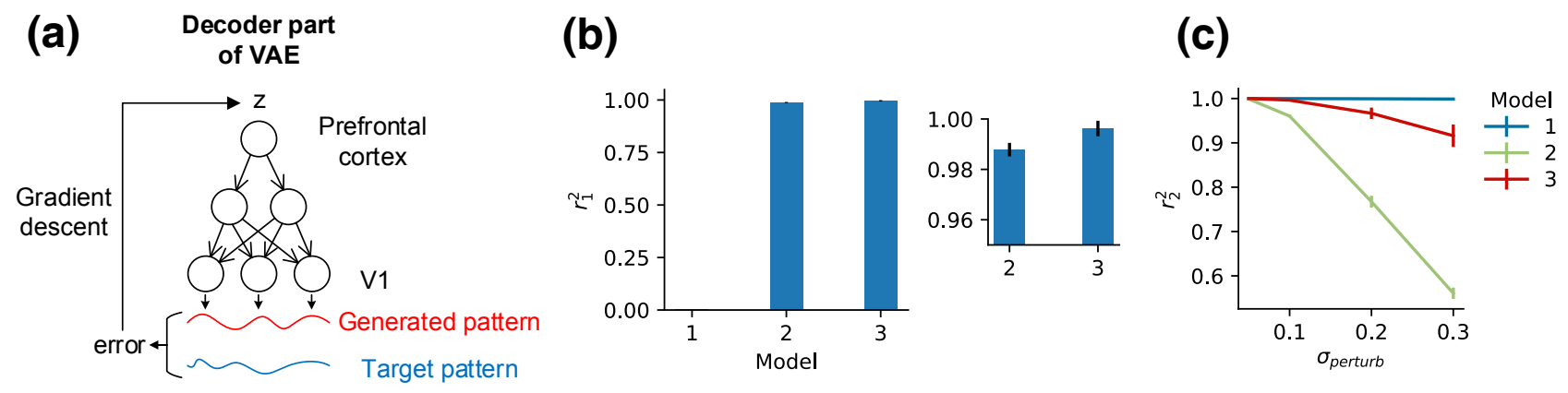

Figure 3: VAE is better at perceiving low-precision representations. (a) Schematic of the perception model. After training, only the decoder of VAE is used. The bottleneck state $z$ is updated by gradient descent to minimize the error between the generated pattern (red) and the target pattern (blue). (b) $r_{1}^{2}$ for the VAEs trained by the three models (Figure 1b). Inset: results for Models 2 and 3 only. (c) $r_{2}^{2}$ as a function of $\sigma_{\text {perturb }}$ for the three models. Error bars represent s.e.m. over 6 VAE configurations.

The advantage of Model 3 over Model 2 is closely related to the smooth transition of $z$ to the shape morphing (i.e., the translational movement) of the generated bumps (Figure 2c), because the generation quality is bad around the abrupt change points in Model 2 (blue curve in Figure 2b, lower panel).

In the study above, we input $\mathcal{P}_{a}$ to VAE and trained VAE to generate $\mathcal{P}_{a}$ in the output, with $\mathcal{P}_{a}$ being the activity pattern of Model $a$ $(a=1,2,3)$. Now we ask whether the advantage of Model 3 results from a 'good' pattern that higher-order cortex receives from V1, or from a 'good' pattern that higher-order cortex is to generate through the top-down pathway. To answer this question, we input $\mathcal{P}_{a}$ to VAE but trained VAE to generate $\mathcal{P}_{b}(b \neq a)$. We found that the quality $r^{2}$ of the generated images strongly depended on $\mathcal{P}_{b}$, but hardly on $\mathcal{P}_{a}$ (Figure 2d). Therefore, the advantage of low-precision representations is a top-down effect, and cannot be understood from a bottom-up perspective.

To understand the reason for the advantage of Model 3, we studied the Euclidean distance $d(s(x), s(y))$ between the activity patterns $s(x)$ and $s(y)$ as a function of $|x-y|$, where $s(x)$ and $s(y)$ are respectively the activity patterns stimulated by two dot stimuli on the 1-dimensional line at positions $x$ and $y$. In Model 1, $d(s(x), s(y))$ sharply jumps from 0 to a constant value at $|x-y|=0$; in Model 2, $d(s(x), s(y))$ is not monotonic; and in Model 3, $d(s(x), s(y))$ monotonically and gradually increases with $|x-y|$ (Figure 2e). This property of Model 3 is important for its advantage in top-down generation. To see this, suppose two nearby bottleneck states $z_{1}$ and $z_{2}\left(z_{1} \approx z_{2}\right)$ generate two patterns $s_{1}$ and $s_{2}$, which corresponds to two dot stimuli at positions $x_{1}$ and $x_{2}$ respectively. For illustration, we constrain that $s_{1}$ is fixed during training, and $s_{2}$ changes within the manifold $\{s(x)\}_{x}$. VAE performs stochastic sampling to determine the bottleneck state, so it is possible that the bottleneck state is sampled to be $z_{2}$ when $s_{1}$ is provided as the input to the encoder and the target output of the decoder during training. By doing so, VAE tries to reduce the distance $d\left(s_{1}, s_{2}\right)$ between the output patterns $s_{1}$ and $s_{2}$ that correspond to $z_{1}$ and $z_{2}$ respectively. In Model 3, due to the monotonicity of $d\left(s_{1}, s_{2}\right)$ with $\left|x_{1}-x_{2}\right|$, reducing $d\left(s_{1}, s_{2}\right)$ reduces $\left|x_{1}-x_{2}\right|$. In this way, two nearby bottleneck states $z_{1}$ and $z_{2}$ correspond to two nearby spatial positions $x_{1}$ and $x_{2}$ respectively; in other worlds, the bottleneck state represents the spatial geometry of the stimuli. In Model 2, $d\left(s_{1}, s_{2}\right)$ is not monotonic with $\left|x_{1}-x_{2}\right|$, so reducing $d\left(s_{1}, s_{2}\right)$ does not always reduce $\left|x_{1}-x_{2}\right|$, sometimes instead increases $\left|x_{1}-x_{2}\right|$. In Model $1, d\left(s_{1}, s_{2}\right)$ remains constant when $s_{1} \neq s_{2}$, so VAE has no gradient clue about how to update $s_{2}$ close to $s_{1}$.

\section{Visual perception}

According to the predictive-coding theory of perception [3], higher-order cortex adjusts its state according to the error of its recontruction of the activity of lower-order cortex. In our model, this is performed by adjusting the bottleneck state $z$ to minimize the error between the target pattern and the pattern generated by the VAE decoder after training (Figure 3a).

Two conditions are required for good perception performance: (1) there exists a state $z_{0}$ at which the generated pattern well resembles the target pattern; and (2) the representations of the target patterns in the bottleneck state should be 'smooth' so that starting from a state $z_{1}$ 

available under aCC-BY-NC-ND 4.0 International license.

$\left(z_{1} \neq z_{0}\right)$, the optimal state $z_{0}$ can be reached by error updating using, in our model, gradient descent algorithm (Figure 3a).

Guided by these two understandings, we studied the perception performance of a VAE trained by Model $a(a=1,2,3)$ in two steps:

(1) We set the target pattern $\mathcal{T}$ (i.e., blue curve in Figure 3a) to be an activity pattern of Model $a$, and initialized the bottleneck state to be the value of the $\mu$-channel (see Figure 1a) of the VAE encoder, which is the optimal bottleneck state estimated by the encoder; then we updated the bottleneck state to minimize the error between $\mathcal{T}$ and the decoder output. We denote the bottleneck state at the end of the updating as $\hat{z}_{0}$.

(2) We set the target pattern $\mathcal{T}$ to be the decoder output when the bottleneck state took $\hat{z}_{0}$, and set the initial bottleneck state to be $\hat{z}_{0}+\epsilon$, with $\epsilon$ being a Gaussian perturbation with standard deviation $\sigma_{\text {perturb }}$, and optimized the bottleneck state again.

We quantified the reconstruction quality in the above two steps using $r_{1}^{2}$ and $r_{2}^{2}$, respectively representing the ratio of variance of the target pattern explained by the decoder output at the end of each step. $r_{1}^{2}$ quantifies how optimal the bottleneck state can be to reconstruct an activity pattern of Model $a(a=1,2,3) . r_{2}^{2}$ quantifies how smooth the representations of the output patterns in the bottleneck state can be so that the optimal bottleneck state can be easily found by error updating. We found that Model 3 has the highest $r_{1}^{2}$ value (Figure 3b), and that Model 3 also has higher $r_{2}^{2}$ value than that Model 2 (Figure 3c).

The high $r_{2}^{2}$ value for Model 1 cannot indicate representation smoothness, because the VAE trained by Model 1 fails to generate delta peak patterns (Figure 2a), so the VAE does not establish representations of Model 1 in the bottleneck state. The low $r_{2}^{2}$ value for Model 2 implies the ruggedness of the representations of the wavelets in the bottleneck state, consistent with the fragmentary representations shown in the upper panel of Figure 2b.

The first step above models a typical two-stage process of perception: information is transmitted feedforwardly at the early stage of perception, but processed recurrently at the later stage [7], which reflects the shift of computational demand from responding speed to representation precision. The value of the $\mu$-channel is the fast-estimation of the bottleneck state by the feedforward pathway before refinement. The second step above studies the case when the feedforward fast-estimation is inaccurate, which may be caused by a fast unexpected change of the stimulus. Our results suggest that the perception of low-precision representations (i.e., the activity pattern of Model 3) is more accurate, and can be more quickly adapted to unexpected situations.

\section{Inferring fine-scale information from the activities of low-coding-precision neurons}

The previous subsection suggests that higher-order cortex is better at simulating or reconstructing low-precision representations for imagination or perception. For fine-scale information in images to be imagined or perceived by the brain, fine-scale information must be able to be inferred from the top-down generated low-precision representations. Here we study this coarse-to-fine inference problem.

We consider a population of low-coding-precision neurons (Figure 4a) receiving stimuli from a one-dimensional circle. The feedforward connection from the stimulus receptor at position $\theta_{\text {stim }}$ to the low-coding-precision neuron preferring $\theta_{\text {low }}$ Gaussianly decays with $\mid \theta_{\text {stim }}-$ $\theta_{\text {low }} \mid$. We study the following problem: given an activity pattern $\mathcal{P}_{\text {low }}$ of the low-coding-precision neurons, how to infer the corresponding pattern of the stimulus receptors?

The stimulus receptors in this model have higher coding precision that the neurons in the second layer. By studying the inference of the activities of stimulus receptors from the activities of the neurons in the second layer, we can gain insight into the inference of fine-scale visual information from low-precision representations.

The most straightforward method is to let the inferred stimulus be $\hat{\mathcal{P}}_{\text {stim }}=\boldsymbol{W}_{\text {forward }}^{-1} \mathcal{P}_{\text {low }}$, where $\boldsymbol{W}_{\text {forward }}$ is the feedforward connection matrix from the stimulus receptors to the low-coding-precision neurons. However, this method is not successful (Figure $4 \mathbf{b}$ ): $\boldsymbol{W}_{\text {forward }}$ has very small eigenvalues corresponding to the eigenvectors that encode fine spatial details (Figure $4 \mathbf{c}$ ), so $\boldsymbol{W}_{\text {forward }}^{-1}$ has very large eigenvalues. These large eigenvalues make $\boldsymbol{W}_{\text {forward }}^{-1}$ strongly amplify random noises (which were induced by limited numeric precision in Figure 4b), impairing the inference.

The inference difficulty results from the fact that a group $\mathcal{G}$ of stimulus receptors with similar position preferences give output to overlapping low-coding-precision neurons, so that it is hard to tell which stimulus receptor causes an activity pattern of the low-coding-precision neurons. If the stimulus receptors in $\mathcal{G}$ have sparse activities, so that in the ideal case only a single receptor in $\mathcal{G}$ is active, all the others being silent, then the inference can be achieved by exploiting the sparseness of $\mathcal{G}$, similar to the compressed sensing scenario [30]. 
(a)
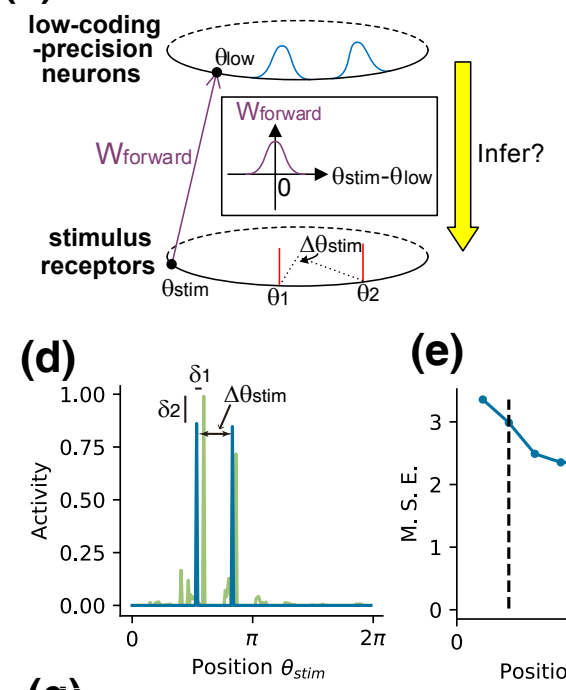

(g)

(e)

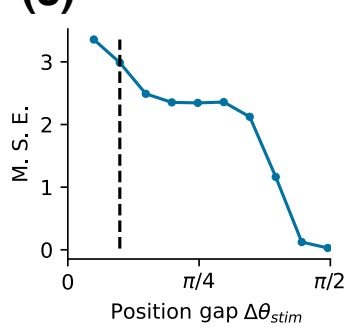

(f)

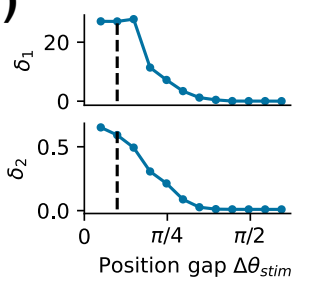

Non-linear act. func.

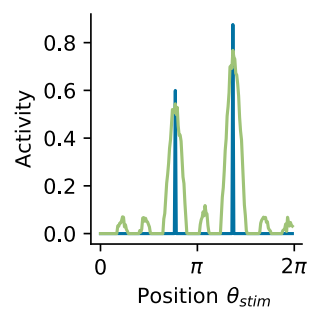

(b)

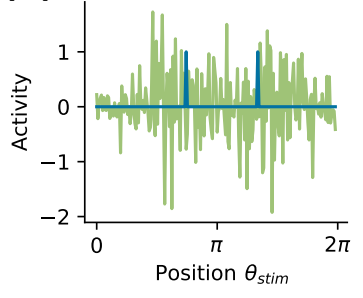

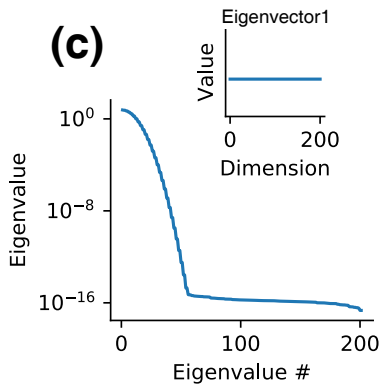

(h)

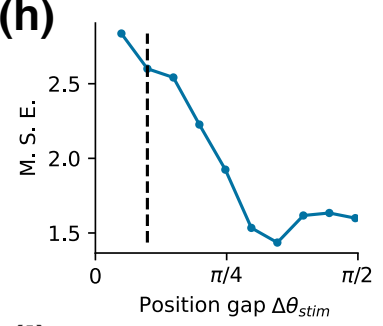

(i)

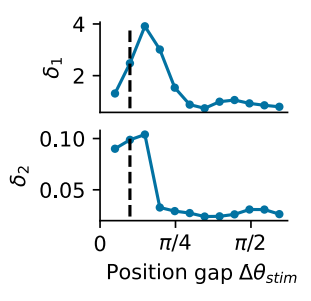

Figure 4: Sparse fine-scale information can be inferred from the activities of low-coding-precision neurons. (a) Schematic of the model. Stimulus receptors and low-coding-precision neurons are located on a circle. The feedforward connections $\boldsymbol{W}_{\text {forward }}$ (magenta) from stimulus receptors to low-coding-precision neurons Gaussianly decays with the difference $\left|\theta_{\text {stim }}-\theta_{\text {low }}\right|$ between their position preferences. The activities of stimulus receptors are delta-peaked (red) at $\theta_{1}$ and $\theta_{2}$ (with $\left.\Delta \theta_{\text {stim }}=\left|\theta_{1}-\theta_{2}\right|\right)$. The activities of low-coding-precision neurons are Gaussianly bumped (blue). We study the inference of the activities of the stimulus receptors from the activities of the low-coding-precision neurons (yellow arrow). (b) Blue: the activity of stimulus receptor as a function of $\theta_{\text {stim }}$. Green: inferred activity using $\boldsymbol{W}_{\text {forward }}^{-1}$. (c) Eigenspectrum of $\boldsymbol{W}_{\text {forward }}$. Insets are examples of eigenvectors. (d) The original (blue) and inferred activities (green) of the stimulus receptors using matching-pursuit algorithm. $\delta_{1}$ and $\delta_{2}$ respectively represent the differences of the positions and heights of the original and inferred peaks. (e) Mean-square error between the original and inferred activities as a function of the gap $\Delta \theta_{\text {stim }}$ between the positions of the two peaks. Vertical dashed line is the standard deviation of the Gaussian decay in $\boldsymbol{W}_{\text {forward }}$. (f) $\delta_{1}$ and $\delta_{2}$ as functions of $\Delta \theta_{\text {stim }}$. (g) Flow chart of the set of operations that can be fulfilled using a network with feedforward and recurrent connections. Three panels below respectively illustrate the original (blue) and inferred (green) activity pattern of the stimulus receptors after each of the three steps. Note that local winner-take-all (WTA) sharpens the peaks, nonlinear activation function suppresses small peaks and also makes the heights the inferred peaks closer to the original ones. (h, i) Similar to panels $\mathbf{e}$ and $\mathbf{f}$, but for the set of operations manifested in panel $\mathbf{g}$. In panels $\mathbf{e}, \mathbf{f}, \mathbf{h}$, $\mathbf{i}$, error bars representing s.e.m. over 8192 samples are smaller than the plot markers. 

available under aCC-BY-NC-ND 4.0 International license.

To manifest the advantage of the sparseness of $\mathcal{G}$ for inference, we supposed that all stimulus receptors except those preferring two positions $\theta_{1}$ and $\theta_{2}$ were silent, and studied how the inference quality depended on $\Delta \theta_{\text {stim }}=\left|\theta_{1}-\theta_{2}\right|$ (Figure $\mathbf{4 a}$ ). When $\Delta \theta_{\text {stim }}$ is small, these two stimulus receptors have similar position preferences, so they can be considered to belong to the same group $\mathcal{G}$; when $\Delta \theta_{\text {stim }}$ gets larger, these two stimulus receptors have farther different preferences, so the group $\mathcal{G}$ which contain stimulus receptors with similar position preferences have sparser activity.

We studied two inference algorithms: (1) a classical matching-pursuit algorithm [31], which aims to explain a given pattern of low-codingprecision neurons using as few active stimulus receptors as possible; (2) a set of operations that can be fulfilled using neural networks with only feedforward and recurrent connections, consisting of a linear transformation, a local winner-take-all operation, and a nonlinear activation function (Figure 4g, see Methods). We used three indexes to quantify the inference quality: (1) mean-square error of the inferred stimulus-receptor activity pattern $\hat{\mathcal{P}}_{\text {stim }}$ from the original pattern $\mathcal{P}_{\text {stim }}$, (2) the difference $\delta_{1}$ between the positions of the peaks in $\hat{\mathcal{P}}_{\text {stim }}$ and $\mathcal{P}_{\text {stim }}$, and (3) the difference $\delta_{2}$ between the heights of the peaks in $\hat{\mathcal{P}}_{\text {stim }}$ and $\mathcal{P}_{\text {stim }}$ (Figure 4d). In both algorithms, all the three indexes indicated that the inference error was small when $\Delta \theta_{\text {stim }}$ was large (Figure 4e,f,h,i). These results suggest that if fine-scale information is sparse, then fine-scale information can be inferred from low-coding-precision neurons. In particular, those fine-scale features that cause similar activity patterns of low-coding-precision neurons should have sparse strength.

\section{Improving imagination and perception of fine-scale information using low-precision generation}

The previous subsections show that the brain is good at generating low-precision representations through top-down pathway, and that finescale information can be inferred from low-precision representations if fine-scale information is sparse. In this subsection, we will show that generating low-precision (instead of high-precision) representations that contain sparse fine-scale information improves imaginative creativity as well as perceptive accuracy and robustness of fine-scale information.

\section{Model setup}

We conducted our study using skeleton MNIST dataset [32]. Images in this dataset (denoted as dataset $\mathcal{D} \mathcal{S}_{1}$ below) represent digits using lines of 1 pixel width (Figure 5a, second column). There are two reasons why we study $\mathcal{D} \mathcal{S}_{1}$. First, images in $\mathcal{D} \mathcal{S}_{1}$ are made up of 1-pixelwidth lines, which are the fine-scale information we would like to study. Second, images in $\mathcal{D} \mathcal{S}_{1}$ are analogous to the activity patterns of V1 simple cells: the thin lines in $\mathcal{D} \mathcal{S}_{1}$ mimic the enhanced representation of contours in V1 due to collinear facilitation [33], whose saliency attracts attention; the thin lines in $\mathcal{D} \mathcal{S}_{1}$ also mimic the sharpened tuning of V1 simple cells under attention [34]. To study the best way to imagine or perceive these 1-pixel-width lines, we trained VAEs using three datasets $\mathcal{D S} \mathcal{S}_{1}, \mathcal{D S}_{2}$ and $\mathcal{D} \mathcal{S}_{3}$, explained below:

(1) $\mathcal{D S} \mathcal{S}_{1}$ can be regarded as the displacement of Model 1 (Figure 1b, left) for 2-dimensional-image case, because a 1-pixel-width line is analogous to the delta-peak pattern in Model 1 along the direction perpendicular to the line (comparing the second column of Figure 5a with Figure 1b, left).

(2) $\mathcal{D S} \mathcal{S}_{2}$ is obtained by processing $\mathcal{D S} \mathcal{S}_{1}$, so that the pixel intensities oscillatorily decay along the direction perpendicular to a line in the skeleton MNIST images (Figure 5a, third column). $\mathcal{D} \mathcal{S}_{2}$ can be regarded as the displacement of Model 2 (Figure 1b, middle) for 2 dimensional-image case.

(3) $\mathcal{D} \mathcal{S}_{3}$ is obtained by processing $\mathcal{D} \mathcal{S}_{1}$, so that the 1-pixel-width lines are blurred, with the pixel intensities Gaussianly decaying along the direction perpendicular to a line (Figure 5a, fourth column). $\mathcal{D S} \mathcal{S}_{3}$ can be regarded as the displacement of Model 3 (Figure 1b, right) for 2-dimensional-image case.

To infer the 1-pixel-width lines from the VAE-generated images, we post-processed the VAE-generated images in the following way: we first binarized the images using a threshold $\theta_{\text {thres }}$, such that pixels with intensities larger (or smaller) than $\theta_{\text {thres }}$ were set to 1 (or 0), then skeletonized the images (see Methods), resulting in binary images with lines of 1-pixel width (Figure $\mathbf{5 b}$ and lower panels of c). By comparing the quality of the post-processed images resulting from the three datasets $\left(\mathcal{D S}, \mathcal{D S}_{2}\right.$ and $\mathcal{D S}$ ), we can know the best way to imagine or perceive the fine-scale information of 1-pixel-width lines. 


\section{(a)}

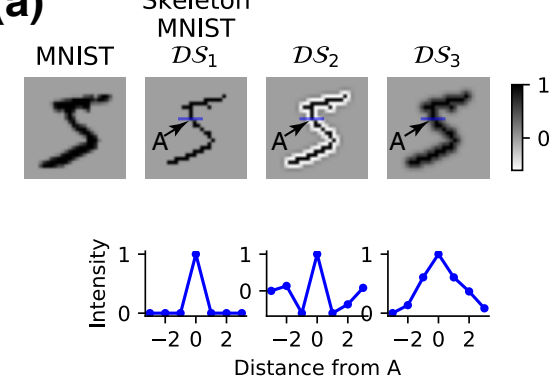

(c)

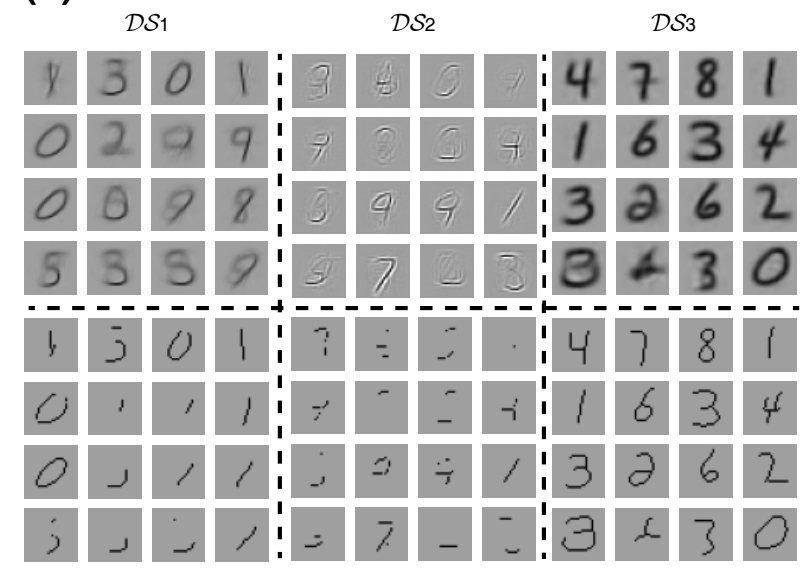

(g)

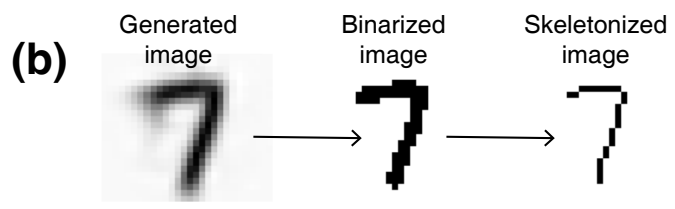

(d)

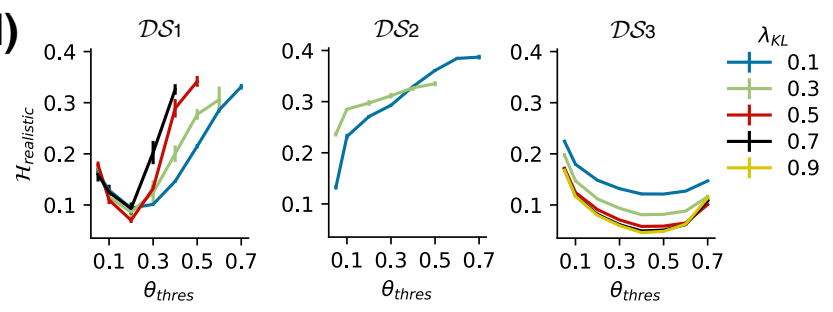

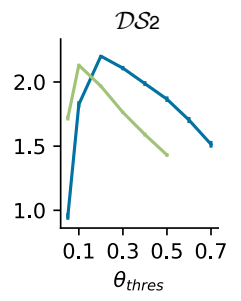

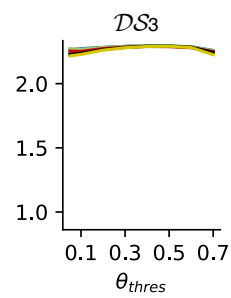

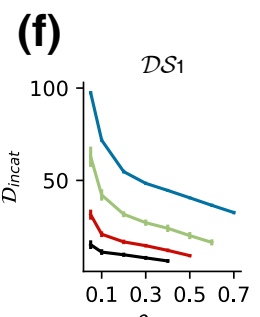

$\theta_{\text {thres }}$

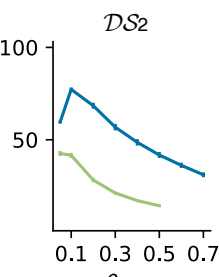

$\theta_{\text {thres }}$

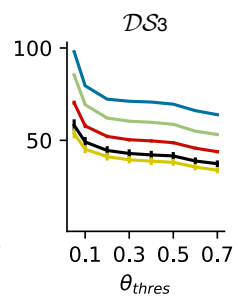

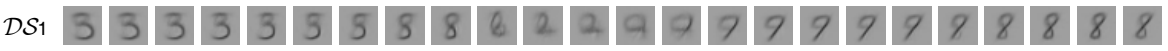

$\mathcal{D S} 2$

${ }_{D S B} 777779444466555555533333$

Figure 5: Low-precision generation facilitates imaginative creativity of fine-scale information. (a) Upper panels: example images in the MNIST, skeleton MNIST $\left(\mathcal{D S}_{1}\right), \mathcal{D S}_{2}$ and $\mathcal{D} \mathcal{S}_{3}$ datasets. Lower panels: pixel intensity along the blue bar in the upper panel as a function of the distance from point $A$ (i.e., the middle point of the bar). (b) An example manifesting the postprocessing of the images generated by VAE. (c) Upper panels: examples of the generated images when using datasets $\mathcal{D} \mathcal{S}_{1}$, $\mathcal{D} \mathcal{S}_{2}$ and $\mathcal{D S} \mathcal{S}_{3}$ to train VAE. Lower panels: the generated images after post-processing. Note that $\mathcal{D S} \mathcal{S}_{3}$ results in better image quality than $\mathcal{D S} \mathcal{S}_{1}$ and $\mathcal{D S} \mathcal{S}_{2}$. (d) $\mathcal{H}_{\text {realistic }}$ as a function of the binarization threshold $\theta_{\text {thres }}$ when the parameter $\lambda_{K L}$ in VAE (see Methods) takes different values, when VAE is trained using $\mathcal{D S} \mathcal{S}_{1}, \mathcal{D S} \mathcal{S}_{2}$ and $\mathcal{D S} \mathcal{S}_{3}$ respectively. Some data points for $\mathcal{D S} \mathcal{S}_{1}$ and $\mathcal{D} \mathcal{S}_{2}$ are missing, see Methods for explanation. (e, f) Similar to panel d, but for $\mathcal{H}_{\text {xcat }}$ and $D_{\text {incat }}$. (g) Examples of the generated images when the bottleneck state slowly and linearly changes its value, see more examples in Supplementary Figure 1. In panels d, e, f, error bars representing s.e.m. over 8 VAE configurations. In panel $\mathbf{c}, \theta_{\text {thres }}=0.4$. In panels $\mathbf{c}$ and $\mathbf{g}, \lambda_{K L}=0.7$ for $\mathcal{D S}_{1}$ and $\mathcal{D S} \mathcal{S}_{3}, \lambda_{K L}=0.3$ for $\mathcal{D S}_{2}$. 

available under aCC-BY-NC-ND 4.0 International license.

As we mentioned in the last subsection (Figure 4), sparseness is required for the inference of fine-scale information from low-precision representations. The inference of 1-pixel-width lines above is also based on the sparseness of 1-pixel-width lines. To understand this point, suppose in a VAE-generated image, there is a very thick line, whose thickness is twice as much as normal. Despite its thickness, however, only a single 1-pixel-width line (instead of two parallel 1-pixel-width lines) results from the skeletonization process illustrated in Figure 5b. Therefore, the inference of 1-pixel-width line by skeletonization is based on the sparseness assumption, and is inaccurate if 1-pixel-width lines are densely aggregated together.

\section{Imaginative creativity}

People have proposed that creativity is a mental process of generating worthwhile and novel ideas [35]. Inspired by this definition of creativity, we propose that good generation of digital images of 1-pixel-width lines should satisfy three conditions. (1) Realisticity: a generated image should look like a digit in dataset $\mathcal{D S}_{1}$. (2) Cross-category variety: the numbers of the generated images looking like different digits should be almost the same. In other words, it is not good if the VAE can generate images looking like the same digit. (3) Within-category variety: the shapes of the images of the same digit should be various. To quantify the image-generation quality, we trained a neural network to classify the images in $\mathcal{D} \mathcal{S}_{1}$, so that the network output a label distribution $p(x \mid I)(x=0,1, \cdots, 9)$ after receiving a post-processed generated image $I$ (see Methods). Realisticity requires that $p(x \mid I)$ has low entropy $\mathcal{H}_{\text {realistic }}$ for each $I$ [36]. Cross-category variety requires that the marginal $\int_{I \in A} p(x \mid I) \mathrm{d} I$ has high entropy $\mathcal{H}_{x c a t}$, with $A$ being the set of all the post-processed generated images [36]. To quantify within-category variety, we calculated the intrinsic dimensionality $D_{\text {incat }}=\frac{\left(\sum_{i} \lambda_{i}\right)^{2}}{\sum_{i} \lambda_{i}^{2}}$ [37], where $\lambda_{i}$ is the eigenspectrum of the post-processed generated images $A_{0}$ belonging to the same category. $D_{\text {incat }}$ has maximal value if all principal components (PCs) of the set $A_{0}$ have equal variance, and has small value if a single PC dominates.

We investigated $\mathcal{H}_{\text {real }}, \mathcal{H}_{x c a t}$ and $D_{\text {incat }}$ with the change of the binarization threshold $\theta_{\text {thres }}$ (Figure 5b) and a parameter $\lambda_{K L}$ in VAE which controls the regularization strength onto the distribution of the bottleneck state variable (see Methods). We found that VAEs trained by $\mathcal{D} \mathcal{S}_{3}$ generated images with better eye-looking quality than VAEs trained by $\mathcal{D} \mathcal{S}_{1}$ or $\mathcal{D} \mathcal{S}_{2}$ (Figure 5c), consistent with the quantitive indications of smaller $\mathcal{H}_{\text {real }}$, larger $\mathcal{H}_{x c a t}$ and larger $D_{\text {incat }}$ of the post-processed images in large range of parameters $\theta_{\text {thres }}$ and $\lambda_{K L}$ (Figure 5d-f, Supplementary Figure 2). These results suggest that generating low-precision representations facilitates the visual system to imagine fine-scale information.

Similar to the 1-dimensional-image case (Figure 2a-c), we also investigated the series of the generated images when the bottleneck state $z$ was continuously changing (Figure 5g). The images generated by the VAE trained by $\mathcal{D} \mathcal{S}_{3}$ have two advantages comparing with those resulting from $\mathcal{D} \mathcal{S}_{1}$ and $\mathcal{D} \mathcal{S}_{2}$ : (1) when the change of $z$ is not large so that the generated images look like the same digit, the images generated by $\mathcal{D} \mathcal{S}_{3}$ undergo more smooth and flexible shape morphing, whereas the images generated by $\mathcal{D} \mathcal{S}_{1}$ and $\mathcal{D} \mathcal{S}_{2}$ are more rigid; (2) when the change of $z$ is large so that the generated images experience digit transition, the images generated by $\mathcal{D} \mathcal{S}_{3}$ look more realistic during the transition period, whereas the images generated by $\mathcal{D} \mathcal{S}_{1}$ and $\mathcal{D} \mathcal{S}_{2}$ are more unrecognizable during the transition period (see Supplementary Figure 1 for more examples). This investigation gains insight into the facilitation of imaginative creativity by low-precision generation.

\section{Visual perception}

To model the perception of an image $I_{1}$ in $\mathcal{D} \mathcal{S}_{1}$, we updated the bottleneck state of a VAE to minimize the difference between the generated image $I_{g}$ (Figure 6a, second row) and the target image $I_{t}$ (Figure 6a, first row), with $I_{t}$ being $I_{1}$ itself, or the image in $\mathcal{D} \mathcal{S}_{2}$ or $\mathcal{D} \mathcal{S}_{3}$ that corresponds to $I_{1}$, depending on whether the VAE was trained by $\mathcal{D} \mathcal{S}_{1}, \mathcal{D} \mathcal{S}_{2}$ or $\mathcal{D} \mathcal{S}_{3}$. To evaluate the performance of the VAE to perceive $I_{1}$, we post-processed the VAE-generated images by binarizing and skeletonizing to infer the 1-pixel-width lines (Figure 5b), and then compared the post-processed images (Figure 6a, third row) with the original image $I_{1}$ from $\mathcal{D} \mathcal{S}_{1}$ (Figure 6a, red box).

The evaluation of perception was performed in two steps. In the first step, we initialized the bottleneck state of the VAE to be the $\mu$-channel of the encoder output in response to $I_{t}$, and then optimized the bottleneck state to minimize the mean square difference between $I_{t}$ and $I_{g}$. We found that $U$, the difference between the post-processed image and $I_{1}$ (see Methods), was lower for VAE trained by $\mathcal{D} \mathcal{S}_{3}$ than for VAE trained by $\mathcal{D} \mathcal{S}_{1}$ or $\mathcal{D} \mathcal{S}_{2}$ (Figure 6b, Supplementary Figure 3a). In the second step, we perturbed the bottleneck state from the optimal state found in the previous step by a Gaussian noise, and then optimized the bottleneck state again (see Methods). We found that the increase of 

available under aCC-BY-NC-ND 4.0 International license.

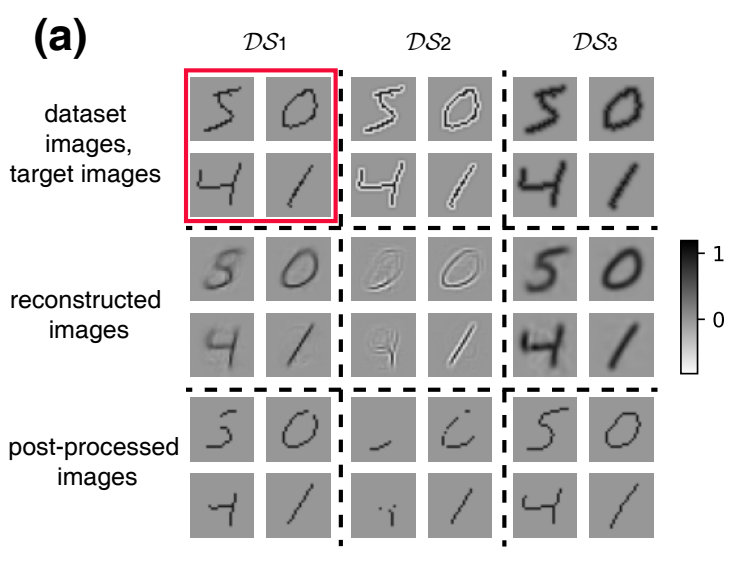

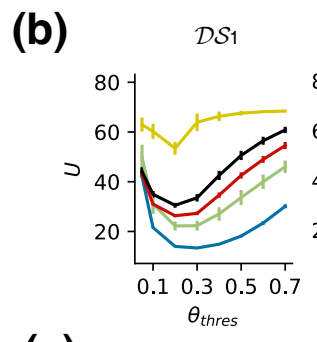

(c)

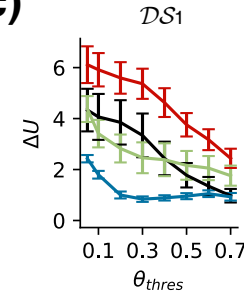

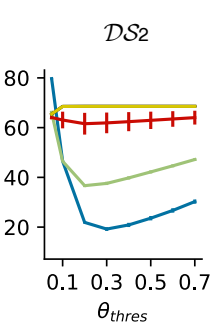

$\mathcal{D S} 2$

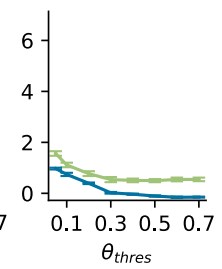

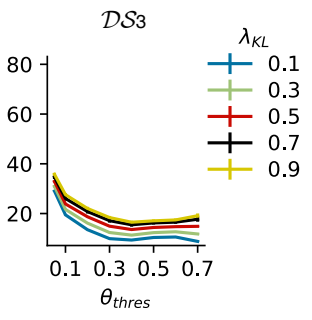

$\mathcal{D S} 3$

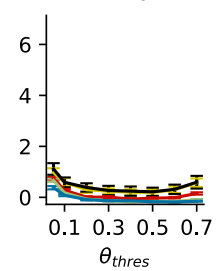

Figure 6: Low-precision generation facilitates perception of fine-scale information. (a) Examples of the dataset images (first row) which are also the target images $I_{t}$ for perception, the reconstructed images $I_{g}$ by VAE (second row), and the images post-processed from the reconstructed images by binarizing and skeletonizing (third row). Note that the post-processed images for $\mathcal{D} \mathcal{S}_{3}$ have the highest similarity to the original images (those in the red box). (b) $U$ as a function of $\theta_{\text {thres }}$ for the three datasets when $\lambda_{K L}$ takes different values. (c) $\Delta U$ is the increase of $U$ value caused by the perturbation of bottleneck state. Some data points for $\mathcal{D} \mathcal{S}_{1}$ and $\mathcal{D} \mathcal{S}_{2}$ are missing, see Methods for explanation. Error bars represent s.e.m. over 8 VAE configurations. In panel a, $\lambda_{K L}=0.3, \theta_{\text {thres }}=0.4$.

$U$ caused by this perturbation was smaller for $\mathcal{D} \mathcal{S}_{3}$ than that for $\mathcal{D S} \mathcal{S}_{1}$ or $\mathcal{D} \mathcal{S}_{2}$ in large parameter range (Figure 6c, Supplementary Figure 3b). Therefore, better and more robust perception of fine-scale information (here, 1-pixel-width lines in $\mathcal{D} \mathcal{S}_{1}$ images) can be achieved by inferring fine-scale information from the reconstruction of low-precision representations (here, $\mathcal{D} \mathcal{S}_{3}$ images).

\section{The imagination and perception of textures}

The digits shown in skeleton MNIST images have a variety of shapes, but lack internal structures. Therefore, we then investigated whether top-down low-precision generation also facilitates the imagination and perception of textures made up of fine-scale information. To this end, we made two databases of textures of 1-pixel-width lines, one synthesized from OpenSimplex2 noise (Figure 7a, $\left.\mathcal{D} \mathcal{S}_{1}\right)$ [38], and the other one from Brodatz database (Supplementary Figure 4) [39], see Methods. Image patches were extracted from each database, processed using similar methods to those shown in Figure 5a, resulting in $\mathcal{D} \mathcal{S}_{1}, \mathcal{D} \mathcal{S}_{2}$ and $\mathcal{D} \mathcal{S}_{3}$ (Figure 7a), which were then used to train VAE. We used image patches of various sizes to train VAE. Large patches contained complicated texture structures (Figure 7a), whereas small patches contained simple short lines (Figure $7 \mathbf{b}$ ).

Imagination was quantified by sliced wasserstein distance (SWD) [40, 41] between the post-processed VAE-generated images (Figure 7c, lower row) and the original images (i.e., $\mathcal{D} \mathcal{S}_{1}$ ). For almost all different image-patch sizes and $\lambda_{K L}$ values we investigated, SWD for $\mathcal{D} \mathcal{S}_{3}$ stays lower than those for $\mathcal{D} \mathcal{S}_{1}$ and $\mathcal{D} \mathcal{S}_{2}$ (Figure 7d, Supplementary Figures 5c, 6a, 7a), which means that the images generated by $\mathcal{D S} \mathcal{S}_{3}$-trained VAE look better like the original images and also have similar variety as the original images (Figure 7c).

Perception accuracy was quantified by the difference $U$ between the post-processed reconstructed images (Figure 7e, 3rd row) and the original images (i.e., $\mathcal{D} \mathcal{S}_{1}$, Figure 7e, red box), with the bottleneck state of VAE before the iterating reconstruction process (Figure 3a) initialized to be the $\mu$-channel of the VAE encoder, see Methods. $U$ for $\mathcal{D} \mathcal{S}_{3}$ is usually lower than that for $\mathcal{D} \mathcal{S}_{1}$ and $\mathcal{D} \mathcal{S}_{2}$, especially for large patch size and high $\lambda_{K L}$ values (Figure 7f, Supplementary Figures 5e, 6b, 7b), which means that generating low-precision images benefits the preception of more complicated textures under stronger constraints on bottleneck state to develop structured image representations [42].

We also studied the robustness of perception by perturbing the bottleneck state optimized in the previous step randomly and then optimizing the bottleneck state again. The increase $\Delta U$ of the perception error caused by this perturbation is most of time close to zero, except for 
(a)

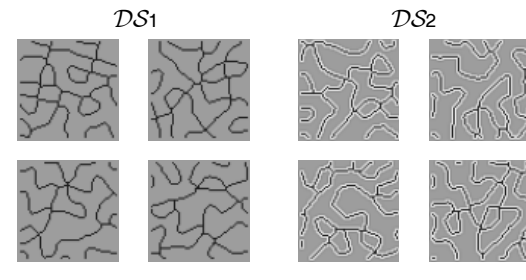

$\mathcal{D S} 3$

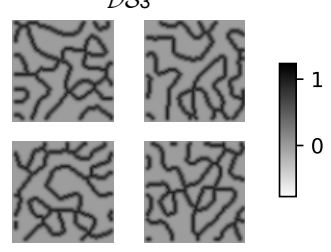

(b)

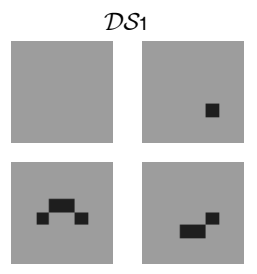

(c)

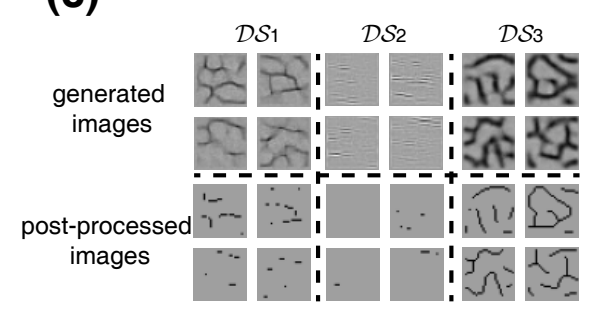

(e)

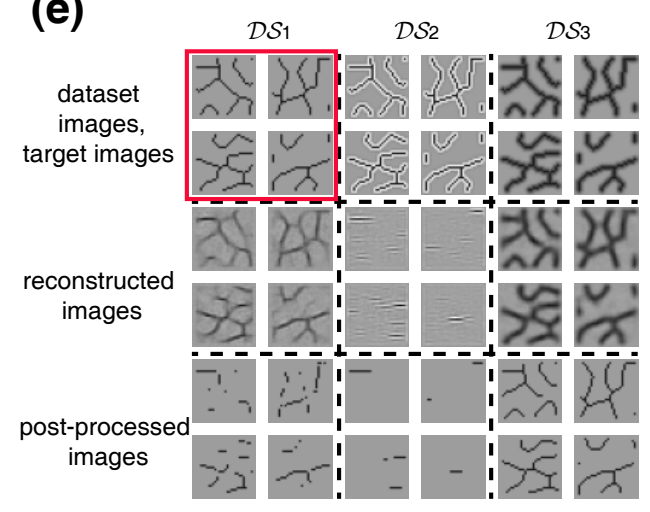

(d)

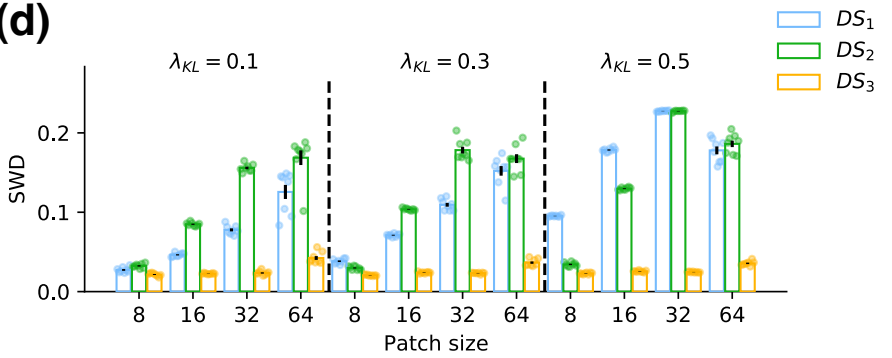

(f)

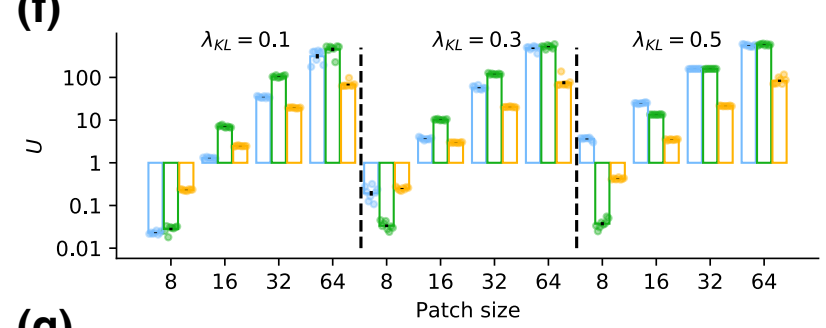

(g)

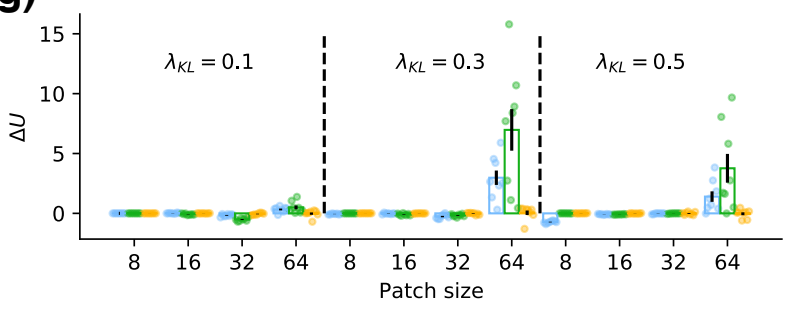

Figure 7: Low-precision generation facilitates imagination and perception of textures. (a) Examples of image patches synthesized from OpenSimplex 2 noise. Image size $64 \times 64$. (b) Examples of image patches with size $8 \times 8$. In $\mathcal{D} \mathcal{S}_{1}$ of both panels $\mathbf{a}$ and $\mathbf{b}$, the intensities of the pixels with distance 1 or 2 from the boundaries were set to zero, see Methods for details. (c) Examples of VAE-generated images (upper row) and post-processed images (lower row). Image size $32 \times 32$. Note that $\mathcal{D} \mathcal{S}_{3}$ result in the best imagination quality. (d) SWD as a function of image-patch size and $\lambda_{K L}$. (e) Examples of targeted images for perception (first row), reconstructed images (second row) and post-processed reconstructed images (third row). Image size $32 \times 32$. Note that $\mathcal{D S} \mathcal{S}_{3}$ result in the best perception quality. (f) Perception error $U$ as a function of image-patch size and $\lambda_{K L}$. (g) Increase $\Delta U$ of perception error caused by perturbation of the bottleneck state. In panels $\mathbf{c}$ and $\mathbf{e}, \lambda_{K L}=0.3$. In panels c-g, $\theta_{\text {thres }}=0.5$. Error bars in panels $\mathbf{d}, \mathbf{f}$ and $\mathbf{g}$ represent s.e.m. over 8 VAE configurations. All panels are results on OpenSimplex2 textures, see Supplementary Figures $\mathbf{4}$ and $\mathbf{5}$ for results on Brodatz textures. 
bioRxiv preprint doi: https://doi.org/10.1101/2021.05.07.443208; this version posted August 30, 2021. The copyright holder for this preprint

(which was not certified by peer review) is the author/funder, who has granted bioRxiv a license to display the preprint in perpetuity. It is made available under aCC-BY-NC-ND 4.0 International license.

large patch size, in which case $\Delta U$ for $\mathcal{D} \mathcal{S}_{3}$ is smaller than those for $\mathcal{D} \mathcal{S}_{1}$ and $\mathcal{D} \mathcal{S}_{2}$ (Figure 7g, Supplementary Figures 5f, 6c, 7c), which demonstrates the benefit of low-precision generation for the robustness of perceiving complicated textures. Interestingly, for Brodatz database, $\Delta U$ usually takes negative values (Supplementary Figures 5f, 7c), which means that such perturbation helps to better reconstruct the target images. This phenomenon remains to be understood.

Overall, the previously claimed computational advantages for low-precision generation largely preserves for textures of 1-pixel-width lines: low-precision generation facilitates imagination (for almost the whole parameter range investigated) and perception (for large image-patch size with complicated texture structure and high $\lambda_{K L}$ value). Considering the structured coding representations respecting the geometry of both spatial and abstract knowledge in the brain [43, 44, 45], high $\lambda_{K L}$, which encourages such structured representations in the bottleneck state [42], is the range that we are interested in.

\section{Discussion}

In this paper, we show that top-down generation of low-precision representations improves the perceptive accuracy and robustness as well as imaginative creativity of visual information with fine spatial scale. This improvement results from two reasons: (1) low-precision representations of fine-scale visual information can be better reconstructed or simulated through top-down pathway than high-precision representations, and (2) fine-scale information can be inferred from low-precision representations if the fine-scale information is sparse. These results suggest that the best way in perceiving or imagining sparse fine-scale information is to generate low-precision representations that contain fine-scale information, instead of generating high-precision representations.

Low-coding precision has been found in extra-classical receptive fields of V1 neurons in perception [26] and the internally generated activities in imagination [22, 23]. Considering that sparseness is a basic computational principle of V1 activity [5], and that fine-scale information can be further sparsified by attention-based selection [46, 47], our results suggest that top-down generating low-precision representations in V1 is not a shortcoming, but instead a strategy that the brain uses to improve the perception and imagination of fine-scale information.

\section{Implications of low-precision generation to brain cognition and machine learning}

We have shown that generating low-precision neural representations facilitates the formation of neural representations in high-order cortex that are smooth to shape morphing of fine-scale information (Figures 2c, 5g), which has implications for both brain cognition and machine learning.

Results of both brain and machine learning studies support the importance of such smooth shape-morphing representations for cognition. Smoothness implies that the semantic meaning of the state of the high-order cortex can be interpolated. States of the high-order cortex that fail in this meaningful interpolation may generate unmeaningful representations during imagination, and cannot guide the updating of the state of the high-order cortex during perception (see the fragmentary represention in Figure $\mathbf{2 b}$ and the corresponding inferior performance in imagination and perception). Consistently, it has been found that human brain represents similar items using similar codes [44], and hippocampus activity also represents the geometry of both spatial and abstract knowledge [43]. In machine learning studies, representations that are smooth to shape morphing have been widely observed in neural network models that generate realistic-looking images [27, 48]. Such representations can be used to do algebraic operations on the semantic meanings of the generated images [49], and can also be exploited to design semi-supervised learning algorithms that train discriminators using only a few labeled data [50, 51].

It is well known that VAE, which tries to construct representations respecting the geometric structure of stimuli in the bottleneck state [42], tends to generate blurred images; vanilla auto-encoder, which does not constrain the structure of representations in the bottleneck state, does not have the tendency to output blurred images [52]. Therefore, the low precision of the top-down generated representations may be a price that the brain has to pay for the structured representations of the world in high-order cortex, which are very important for cognition as discussed in the paragraph above.

Engineering neural networks to generate images with high-resolution details is a non-trivial task. VAE tends to generate blurred images [52]. Another well-known generative model, generative adversarial network (GAN), also struggles to generate high-resolution details [53] 

available under aCC-BY-NC-ND 4.0 International license.

due to a gradient problem [54]. Our results suggest that blurring these high-resolution details may result in better imagination performance, as long as these high-resolution details can be later inferred from the generated low-resolution representations. The implementation of this idea in engineering VAE or GAN for generating real-world images is an interesting research direction.

In [22], it is found that the receptive fields of $\mathrm{V} 1$ neurons in imagined images have larger sizes and lower spatial-frequency preferences than in seen images. A direct prediction of our study is that V1 neurons also have less sensitivity to grating phases in imagined images, which may be tested using the experimental protocol of [22].

\section{Implications of $\mathrm{V} 1$ computation}

Sparse coding has long been believed as a computational principle of V1 [5]. Here we show that sparseness is a requirement for finescale information to be inferred from top-down generated low-precision representations. In other words, for fine-scale information to be represented by high-order cortex, it must be sparse. Besides the sparseness of V1 activity [5], sparseness can also be realized by attention. Attention not only selects salient or task-relevant sensory inputs [46, 47], sparsifying the fine-scale information for perception, but also selects features to imagine, sparsifying the features under anticipation or intention before actually sensing the stimuli [55, 56].

Our results also gain understanding of V1 computation during predictive-coding perception. We have shown that better perception results from updating the state of the high-order cortex according to the error calculated from low- (instead of high-) precision representations (Figures 3, 6, 7). Therefore, it is better to first transform the bottom-up stimulated high-precision representations to low precision before calculating the bottom-up and top-down mismatch and transmitting this mismatch feedforwardly for predictive-coding perception. Such high-to-low precision transformation can easily be done through feedforward projections [30]. It has been found that top-down prediction error is feedforwardly transmitted in predominantly high (gamma) frequency range [57, 58], so the coding precision of prediction error may be understood by investigating the activities of V1 neurons in gamma frequency range.

\section{The power-law eigenspectrum of V1 activity}

It has been found that the variance of the $n$th principal component of V1 activity decays in power law $n^{-\alpha}$ with $\alpha \rightarrow 1^{+}$[59]. The value of this exponent $\alpha$ ensures the differentiability of neural code, such that the V1 response smoothly changes with the position of a stimulus, and the distance between the V1 responses to two stimuli increases with the distance between the two stimuli [59]. Our results support the importance of code differentiability for imagination and perception. In Model 3, which results in the best imagination and perception performance in the 1-dimensional-image case (Figures 2,3), the Euclidean distance between two activity patterns gradually and monotonically increases with the distance between the two dot stimuli corresponding to the two patterns (Figure 2e). Similarly, in $\mathcal{D} \mathcal{S}_{3}$, which results in the best performance in the 2-dimensional-image cases (Figures 5, 6, 7), the intensity of a pixel gradually and monotonically decays with the distance of this pixel from the center of a blurred line, such that the Euclidean distance between two textures $\mathcal{T}_{1}$ and $\mathcal{T}_{2}$ gradually and monotonically increases when $\mathcal{T}_{2}$ experiences gradual local shape morphing starting from $\mathcal{T}_{1}$. Therefore, the differentiability of V1 activity [59] may be closely related to the top-down generative function of the visual system, which may be understood as follows. Suppose that higher-order cortex represents a stimulus at position $x$ using firing-rate code $z(x)$, and the V1 firing-rate code is $v_{1}(x)$. Predictive-coding perception and realistic imagination require that $v_{1}(x) \approx f(z(x))$, where $f(\cdot)$ represents the top-down network, which is differentiable in the biologically plausible context. Therefore, if $z(x)$ is to be differentiable to $x$ (which means that higher-order cortex forms representations differentiable to shape morphing of stimuli, see Figure 2c), $v_{1}(x)$ must also be differentiable to $x$ to better fulfill the equation $v_{1}(x) \approx f(z(x))$. 


\section{Methods and Materials} available under aCC-BY-NC-ND 4.0 International license.

\section{The imagination and perception of 1-dimensional images}

\section{VAE structure}

A variational auto-encoder (VAE) [27] contains two parts: an encoder and a decoder (Figure 1a). The encoder is a feedforward network that receives input $\boldsymbol{x}$ and gives two channels of output $\boldsymbol{\mu}$ and $\log \left(\boldsymbol{\sigma}^{2}\right)$. Then a random number $\boldsymbol{z}$ is generated according to the Gaussian distribution $\mathcal{N}(\boldsymbol{\mu}, \boldsymbol{\sigma})$, and is input to the decoder which outputs $\hat{\boldsymbol{x}}$. VAE is trained to minimize the following cost function:

$$
E_{V A E}=\sum_{i=1}^{D_{x}}\left(x_{i}-\hat{x}_{i}\right)^{2}-\frac{\lambda_{K L}}{2} \sum_{j=1}^{D_{z}}\left(1+\log \left(\sigma_{j}^{2}\right)-\mu_{j}^{2}-\sigma_{j}^{2}\right),
$$

where $D_{x}$ is the dimension of the input and the output, $D_{z}$ is the dimension of the random variable $z$. Minimizing the first term of this equation reduces the reconstruction error, minimizing the second term (which is the KL-divergence of $\mathcal{N}(\boldsymbol{\mu}, \boldsymbol{\sigma})$ from the standard normal distribution $\mathcal{N}(\mathbf{0}, \mathbf{1})$ ) makes $\mathcal{N}(\boldsymbol{\mu}, \boldsymbol{\sigma})$ close to $\mathcal{N}(\mathbf{0}, \mathbf{1}) . \lambda_{K L}$ is a parameter controlling the relative strengths of these two terms.

In the VAE used in Figures 2 and 3, the encoder is a multilayer perceptron (MLP) that has three hidden layers with sizes 100, 50 and 20 respectively. The input layer is of size 201, and the output layer has two channels each with size 1 . The decoder is another MLP that has three hidden layers with sizes 20,50 and 100 respectively. Adjacent layers are all-to-all connected. We use leaky relu as the activation functions of the hidden layers. VAE was trained using Adam optimizer [60] using Pytorch.

\section{The three models of 1-dimensional images}

In the three models in Figure 1b, 201 neurons are positioned on a one-dimensional line. In Model 1, the activity pattern is a delta peak $f_{1}(x ; a)=\delta_{x, a}$ in which only a single neuron at position $a$ has activity 1 , whereas all the other neurons have zero activity. In Model 2 , this pattern is a Gabor wavelet $f_{2}(x ; a)=C \exp \left(-\frac{(x-a)^{2}}{2 \sigma^{2}}\right) \sin \left(\frac{2 \pi}{T}(x-a)\right)$, where $\sigma=10, T=80, C$ is a normalization factor such that $\max _{x} f_{2}(x ; a)=1$. In Model 3, this pattern is a Gaussian bump $f_{3}(x ; a)=C \exp \left(-\frac{(x-a)^{2}}{2 \sigma^{2}}\right)$, where $\sigma=10, C$ is a normalization factor such as $\max _{x} f_{3}(x ; a)=1$. In $f_{1}(x ; a), f_{2}(x ; a)$ and $f_{3}(x ; a), a$ is a random integer from 31 to 171 .

\section{The quality of imagination}

To quantify the quality of the generated patterns (Figure 2d), for any generated pattern $p$, we defined $r^{2}(p)=\max _{x}\left(r_{0}^{2}(p, s(x))\right)$, where $s(x)$ is the activity pattern of Model-out in response to the dot stimulus at position $x$ on the 1-dimensional line, and $r_{0}^{2}(p, s(x))$ is the ratio of the variance of $p$ that can be explained by $s(x)$ (i.e., coefficient of determination). Specifically, $r_{0}^{2}(p, s)$ is defined by

$$
r_{0}^{2}(p, s)=\frac{\sum_{i}\left(p_{i}-s_{i}\right)^{2}}{\sum_{i}\left(p_{i}-\langle p\rangle\right)^{2}}
$$

where $p_{i}$ and $s_{i}$ are respectively the activities of the $i$ th neuron in the patterns $p$ and $s,\langle p\rangle$ is the mean activity of pattern $p$.

\section{The quality of perception}

In Figure 3, the bottleneck state was optimized to minimize the error between the target pattern and the generated pattern using Adam optimizer [60]. Both $r_{1}^{2}$ and $r_{2}^{2}$ are the coefficient of determination to quantify how much variance of the target pattern can be explained by the generated pattern. 

available under aCC-BY-NC-ND 4.0 International license.

\section{The imagination and perception of skeleton MNIST images}

\section{VAE structure}

The VAE used in Figures 5 and 6 has a similar structure with that used in Figures 1-3, except that the size of the input and output layers is $28 \times 28=784$, and the sizes of the three hidden layers of the encoder (or decoder) are 512, 256 and 128 (or 128, 256 and 512) respectively. The size of each of the two output channels of the encoder is 20 .

\section{The three datasets of 2-dimensional images}

The dataset $\mathcal{D S} \mathcal{S}_{1}$ in Figure 5a is the skeleton MNIST dataset [32]. The intensity of a pixel in an image in $\mathcal{D} \mathcal{S}_{1}$ is 1 or 0 depending on whether this pixel belongs to a line of 1-pixel width.

An image $I_{2}$ in $\mathcal{D} \mathcal{S}_{2}$ was obtained using an image $I_{1}$ in $\mathcal{D} \mathcal{S}_{1}$ in the following way. To determine the intensity $T_{2}\left(x_{2}, y_{2}\right)$ of a pixel $I_{2}\left(x_{2}, y_{2}\right)$ at the position $\left(x_{2}, y_{2}\right)$ in $I_{2}$, we defined a box $\mathcal{B}\left(x_{2}, y_{2}\right)=\left\{I_{1}(\eta, \xi):\left|\eta-x_{2}\right| \leq 2,\left|\xi-y_{2}\right| \leq 2\right\}$ in $I_{1}$. We looked for a pixel $I_{1}\left(x_{1}, y_{1}\right) \in \mathcal{B}\left(x_{2}, y_{2}\right)$ such that its intensity $T_{1}\left(x_{1}, y_{1}\right)=1$ and the distance $d=\sqrt{\left(x_{1}-x_{2}\right)^{2}+\left(y_{1}-y_{2}\right)^{2}}$ was minimized. Then we set $T_{2}\left(x_{2}, y_{2}\right)=a \exp \left(-d^{2} / 2\right)$, where $a=-1$ if $\max \left(\left|x_{1}-x_{2}\right|,\left|y_{1}-y_{2}\right|\right)=1$, and $a=1$ otherwise. If all pixels in $\mathcal{B}\left(x_{2}, y_{2}\right)$ had intensity 0 , then $T_{2}\left(x_{2}, y_{2}\right)=0$.

$\mathcal{D} \mathcal{S}_{3}$ was generated using $\mathcal{D S} \mathcal{S}_{1}$ in a similar way to above, except that $a=1$ all the time.

\section{The quality of imaginative creativity}

The images generated by VAE were post-processed in two steps (Figure 5b). First, images were binarized such that pixels with intensities larger (or smaller) than a threshold $\theta_{\text {thres }}$ were set to 1 (or 0). Second, the images were skeletonized using the 'skeletonize' routine of the skimage python package.

To quantify the quality of the post-processed images, we trained a MLP to classify the skeleton MNIST dataset (i.e., $\mathcal{D} \mathcal{S}_{1}$ ). This MLP contained a hidden layer of size 1000 with leaky-relu activation function. After receiving a post-processed VAE-generated image $I$, this MLP output a label distribution $p(x \mid I)(x=0,1, \cdots, 9)$. In Figure 5d, $\mathcal{H}_{\text {realistic }}=E_{I}\left[-\sum_{x} p(x \mid I) \ln p(x \mid I)\right]$, where $E_{I}[\cdot]$ means average over all post-processed VAE-generated images [36]; in Figure 5e, $\mathcal{H}_{x c a t}=-\sum_{x} E_{I}[p(x \mid I)] \ln E_{I}[p(x \mid I)]$ [36]. To plot Figure 5f, we first chose the post-processed VAE-generated images with high realisticity (i.e., $\max _{x} p(x \mid I)>0.9$ ), then for all the images belonging to a category $x$, we calculated the variance $\lambda_{i}(x)$ along the $i$ th principal component (PC), $D_{\text {incat }}$ was defined as $D_{\text {incat }}=E_{x}\left[\frac{\left(\sum_{i} \lambda_{i}(x)\right)^{2}}{\sum_{i} \lambda_{i}^{2}(x)}\right]$ [37]. Figure 5d-f show how $\mathcal{H}_{\text {realistic }}, \mathcal{H}_{x c a t}$ and $D_{\text {incat }}$ change with the binarization threshold $\theta_{\text {thres }}$ and the parameter $\lambda_{K L}$ in eq. 1. Note that if $\theta_{\text {thres }}$ is high, the image after post-processing may be very sparse (i.e., only a few pixels are nonzero), especially when $\lambda_{K L}$ also takes a large value. In this case, the MLP network has an artifact that $p(x \mid I)$ strongly peaks at $x=1$, and $p(x \neq 1 \mid I)$ has very small value. Because of this artifact, in Figure 5d-f, we excluded the data points at which the percentage of nonzero pixels in the post-processed images was smaller than $1 \%$. Some data points when $\lambda_{K L}=0.9$ for $\mathcal{D} \mathcal{S}_{1}$ and when $\lambda_{K L}=0.5,0.7,0.9$ for $\mathcal{D} \mathcal{S}_{2}$ resulted in images with sparsity a little larger than $1 \%$, but we also excluded these data points, because the quality of the generated images was really bad. This artifact was weak for $\mathcal{D S} \mathcal{S}_{3}$ in our range of parameters, so we plotted the whole parameter range for $\mathcal{D} \mathcal{S}_{3}$.

Figure $5 \mathbf{g}$ was plotted by gradually and linearly changing the bottleneck states of VAEs from $\boldsymbol{z}=[1.5,1.5, \cdots, 1.5]$ to $[-1.5,-1.5, \cdots,-1.5]$.

\section{The quality of perception}

The performance of a VAE to perceive a skeleton MNIST image was quantified in the following two steps:

(1) Given an image $I_{\text {origin }}$ in $\mathcal{D} \mathcal{S}_{1}$, we let the target image $I_{t}$ be $I_{\text {origin }}$ itself or the corresponding image in $\mathcal{D} \mathcal{S}_{2}$ or $\mathcal{D} \mathcal{S}_{3}$, depending on whether the VAE was trained by $\mathcal{D} \mathcal{S}_{1}, \mathcal{D} \mathcal{S}_{2}$ or $\mathcal{D} \mathcal{S}_{3}$. We set the initial bottleneck state to be the $\mu$-channel of the VAE encoder in response 
to $I_{t}$, and optimized the bottleneck state to minimize the mean square error between $I_{t}$ and the VAE-generated image $I_{g}$. $I_{g}$ was then post-processed by binarizing and skeletonizing, resulting in image $I_{\text {postprocess }}$. The perception quality was then quantified by (Figure $6 \mathbf{b}$ )

$$
U=\left\langle\sum_{i}\left(\operatorname{Blur}\left(I_{\text {origin }}\right)_{i}-\operatorname{Blur}\left(I_{\text {postprocess }}\right)_{i}\right)^{2}\right\rangle_{I_{\text {origin }}}
$$

where both $I_{\text {postprocess }}$ and $I_{\text {origin }}$ are made up of 1-pixel-width lines, and Blur $(\cdot)_{i}$ means the $i$ th pixel of a blurred image. This blurring operation was performed using the same method as we produced $\mathcal{D} \mathcal{S}_{3}$ from $\mathcal{D} \mathcal{S}_{1}$. To understand the reason for this blurring operation, suppose there are two 1-pixel-width lines taking the same direction in $I_{\text {origin }}$ and $I_{\text {postprocess }}$ respectively. In this case, $\sum_{i}\left(I_{\text {origin }, i}-\right.$ $\left.I_{\text {postprocess }, i}\right)^{2}$ is zero if these two parallel lines overlap, whereas takes the same positive value if these two parallel lines do not overlap, no matter whether they are located spatially nearby or far apart. By blurring $I_{\text {origin }}$ and $I_{\text {postprocess }}, U$ gradually increases with the distance between these two parallel lines, which is consistent with the intuition that the perception error increases with the distance between these two lines.

(2) We then perturbed the optimized bottleneck state in the above step by a Gaussian noise with standard deviation $\sigma_{\text {perturb }}=0.4$, optimized the bottleneck state again to minimize the difference between $I_{g}$ and $I_{t}$, and then calculated $U$ using eq. 3 again. Figure $6 \mathbf{c}$ plots the increase of $U$ value in this second step from that in the first step. Some data points for $\mathcal{D} \mathcal{S}_{1}$ (when $\left.\lambda_{K L}=0.9\right)$ and $\mathcal{D} \mathcal{S}_{2}\left(\right.$ when $\lambda_{K L}=0.5,0.7,0.9$ ) are missing in Figure 6c and Supplementary Figure 3b. The reason is that for $\mathcal{D} \mathcal{S}_{1}$ and $\mathcal{D} \mathcal{S}_{2}$, the reconstruction quality is so bad when $\lambda_{K L}$ takes large values, such that the perception error $U$ already takes large values (Figure 6b). In this case, noise perturbation can hardly increase $U$ any more, which makes $\Delta U$ close to zero. However, this close-to-zero $\Delta U$ does not imply perception robustness, but is instead an artifact in the case of very bad perception. To avoid possible confusion of the readers and let $\Delta U$ faithfully represent perception robustness, we deleted these data points in Figure 6c and Supplementary Figure 3b.

\section{The imagination and preception of texture images}

\section{Database preparation}

In Figure 7 and Supplementary Figures 4-7, we studied the generation of textures using two databases: one synthesized from OpenSimplex2 noise [38], and the other from Brodatz database [39].

The OpenSimplex2 textures were generated using FastNoiseLite package with the following parameters. Noise type: OpenSimplex2; frequency: 0.05; fractal type: ridged; fractal octaves: 1; fractal lacunarity: -0.29 ; fractal gain: 0.05; fractal weighted strength: 1.3; domain warp type: OpenSimplex2; domain warp amplitude: 5 . The generated images were then binaried through threshold 0.5 , and skeletonized by the 'skeletonize' routine of skimage python package, resulting in 60000 images of size $64 \times 64$.

The Brodatz textures were generated by treating normalized Brodatz's textures [39] using the command '1-edge(imgaussfilt(data, 2), 'Canny')' of Matlab, binarizing through threshold 0.5, and then skeletonizing, resulting in 112 images of size $640 \times 640$.

\section{Image patch preparation}

We extracted image patches of different sizes $(8 \times 8,16 \times 16,32 \times 32$ and $64 \times 64)$ at random positions from the two databases respectively. For each image patch, we set the intensities of the pixels whose distances with boundaries were 1 or 2 to be zero (see below for the reason for this operation), resulting in dataset $\mathcal{D S}$. Datasets $\mathcal{D S} \mathcal{S}_{2}$ and $\mathcal{D} \mathcal{S}_{3}$ were derived from $\mathcal{D} \mathcal{S}_{1}$ in similar ways to those for skeleton MNIST images.

\section{The quality of imagination}

We post-processed the VAE-generated images by binarizing and skeletonizing, and then compared the obtained images of 1-pixel-width lines with the original images in $\mathcal{D} \mathcal{S}_{1}$ to evaluate the imagination quality of VAE. The skeletonization operation (i.e., the 'skeletonize' routine of skimage python package) has an artifact: if both a pixel at the boundary of an image and a nearby pixel not at the boundary take 1 

available under aCC-BY-NC-ND 4.0 International license.

value after binarization, then the boundary pixel is more likely to belong to a 1-pixel-width line after skeletonization than the non-boundary pixel. To avoid this boundary artifact, we need to reduce the intensities of the pixels in the VAE-generated images at the boundary, so that they are unlikely to take 1 value after binarization. To this end, as we mentioned before, we set the intensities of the pixels whose distances with boundaries were 1 or 2 to be zero in dataset $\mathcal{D} \mathcal{S}_{1}$, before $\mathcal{D S} \mathcal{S}_{2}$ and $\mathcal{D} \mathcal{S}_{3}$ were derived from $\mathcal{D} \mathcal{S}_{1}$ and used to train VAE.

The imagination quality was quantified using sliced wasserstein distance (SWD) [40, 41] between the post-processed VAE-generated images and $\mathcal{D S}_{1}$. SWD is defined by

$$
\operatorname{SWD}=\left\langle\sum_{j}\left|\operatorname{sort}_{j}\left(\{\boldsymbol{x} \cdot \boldsymbol{n}\}_{\text {batch } \boldsymbol{x}}\right)-\operatorname{sort}_{j}\left(\{\tilde{\boldsymbol{x}} \cdot \boldsymbol{n}\}_{\text {batch } \tilde{\boldsymbol{x}}}\right)\right|\right\rangle_{\boldsymbol{n}},
$$

where $\boldsymbol{x}$ and $\tilde{\boldsymbol{x}}$ are respectively the post-processed VAE-generated images and $\mathcal{D} \mathcal{S}_{1}, \boldsymbol{n}$ is a randomly selected direction vector with $\|\boldsymbol{n}\|^{2}=1$, $\operatorname{sort}_{j}\left(\{\boldsymbol{x} \cdot \boldsymbol{n}\}_{\text {batch } \boldsymbol{x}}\right)$ means the $j$ th element after sorting the value $\boldsymbol{x} \cdot \boldsymbol{n}$ in a batch of $\boldsymbol{x},\langle\cdot\rangle_{\boldsymbol{n}}$ means averaging over $\boldsymbol{n}$. Numerically, the batch sizes of both $\boldsymbol{x}$ and $\tilde{\boldsymbol{x}}$ were 1024, and we averaged over 512 directions of $\boldsymbol{n}$ when calculating SWD.

\section{The quality of perception}

The accuracy and robustness of perception were respectively quantified by $U$ defined in eq. 3 and the increase of $U$ after optimizing the perturbed bottleneck state, in a similar way to that for skeleton MNIST images.

\section{The network structure of VAE}

The VAEs to generate texture images (Figure 7) were deep convolutional neural networks with batch normalization and leaky relu activation function following each convolutional layer. In the encoder part of VAE, convolutional kernels had spatial size 4 and stride 2 , such that the spatial size of signal was reduced by a half after each convolutional layer. In the decoder part, transposed convolutional kernals also had spatial size 4 and stride 2, such that the spatial size of signal was increased by a time after each transposed convolutional layer. For VAEs used to generate images of size $8 \times 8,16 \times 16,32 \times 32$ and $64 \times 64$, there were 2, 3, 4 and 5 convolutional (or transposed convolutional) layers in the encoder (or decoder) respectively. In the 1st, 2nd, 3rd, 4th and 5th convolutional layers in the encoder (or the last 1 st, 2 nd, 3rd, 4th and 5th transposed convolutional layers in the decoder), there were 64, 128, 256, 512 and 512 channels respectively (note that some high-order layers did not exist in the VAE if the image size was smaller than $64 \times 64$ ).

\section{Inferring fine-scale information from the activities of low-coding-precision neurons}

In our simulation for Figure 4a, both the stimulus receptor layer and the low-coding-precision neuron layer contained $N=201$ neurons lying on a one-dimensional circle. Only two stimulus receptors were active, separated by

$$
\Delta N=\Delta \theta_{\text {stim }} N / 2 \pi
$$

neurons, with $\Delta N=5,10,15, \cdots, 60$. The activities of the two active stimulus receptors were set to two random values in the range $[0.5,1]$, and the activities of all the other stimulus receptors were zero.

The feedforward connection from a stimulus receptor preferring $\theta_{\text {stim }} \in[0,2 \pi)$ to a low-coding-precision neuron preferring $\theta_{\text {low }} \in[0,2 \pi)$ was

$$
W_{\text {forward }}\left(\theta_{\text {stim }}, \theta_{\text {low }}\right)=C \exp \left(-\frac{\left(\min \left(2 \pi-\left|\theta_{\text {stim }}-\theta_{\text {low }}\right|,\left|\theta_{\text {stim }}-\theta_{\text {low }}\right|\right)\right)^{2}}{2 \sigma^{2}}\right)
$$

where $\sigma=2 \pi \times 10 / N, C$ is a normalization factor so that $\sqrt{\sum_{n=1}^{201} W_{\text {forward }}\left(\theta_{\text {stim }}, 2 \pi n / N\right)^{2}}=1$.

Given the activities $\left\{x_{1}, x_{2}, \cdots, x_{N}\right\}$ of stimulus receptors, we calculated the activities $\left\{y_{1}, y_{2}, \cdots, y_{N}\right\}$ of low-coding-precision neurons using the feedforward matrix $\boldsymbol{W}_{\text {forward }}$, and then we studied how to infer the activities $\left\{\hat{x}_{1}, \hat{x}_{2}, \cdots, \hat{x}_{N}\right\}$ of stimulus receptors using $\left\{y_{1}, y_{2}, \cdots, y_{N}\right\}$. 
We first tried matching-pursuit algorithm [31] (Figure 4d-f). Specifically, we first defined the remnant $r_{i}=y_{i}$, and set $\hat{x}_{i}=0(i=$ $1,2, \cdots, N)$, then iterated the following steps for 1000 times:

(i) Calculate $c_{i}=\sum_{j=1}^{N} r_{j}\left(W_{\text {forward }}^{T}\right)_{j i}$, then set $c_{k}=0$ for $k \neq \arg \max _{i}\left|c_{i}\right|$.

(ii) Let $\hat{x}_{i} \leftarrow \hat{x}_{i}+c_{i}, r_{i} \leftarrow r_{i}-\sum_{j=1}^{N} c_{j}\left(W_{\text {forward }}\right)_{j i}$.

To manifest the biological possibility of the inference, we then performed this inference using a set of operations that can be fulfilled using neural networks that contain only feedforward and recurrent connections, consisting of a linear transformation, a local winner-take-all (WTA) operation, and a nonlinear activation function (Figure $\mathbf{4 g - i}$ ). The linear transformation was defined by $g_{L, i}=\sum_{j} w_{i j} y_{j}$, where $y_{j}$ is the activity of the $j$ th low-precision neuron, $g_{L, i}$ is the $i$ th output of the linear transformation, $w_{i j}$ is the transformation matrix. We determined $w_{i j}$ by minimizing the following cost function:

$$
E_{L}=\sum_{i}\left(x_{i}+\epsilon\right)\left(g_{L, i}-x_{i}\right)^{2},
$$

where $\epsilon=0.01$. This cost function means that we would like $g_{L, i}$ to be close to $x_{i}$, emphasizing this closeness for large $x_{i}$ values. The output $g_{W, i}$ of the local WTA operation was calculated by initializing $g_{W, i}=g_{L, i}$ and the set $\mathcal{P}=\emptyset$, and then iterating the following steps: selecting the $i \notin \mathcal{P}$ with maximal $g_{W, i}$, let $g_{W, j}=0$ for $0<|j-i| \leq \sigma_{W T A}$ (where $\sigma_{W T A}$ is the spatial scale of the local WTA), and let $\mathcal{P}=\mathcal{P} \cup\{i\}$. In our simulation, we set $\sigma_{W T A}=$ floor $((\Delta N+1) / 2)$, where floor $(x)$ is the maximal integer that is no larger than $x$, and $\Delta N$ is the distance between the two peaks in the activity pattern of the stimulus-receptor layer, defined in eq. $\mathbf{5}$. The output $g_{N, i}$ of the nonlinear activation function was calculated as

$$
g_{N, i}=\left\{\begin{array}{ll}
0, & g_{W, i}<\theta_{t h} \\
a g_{W, i}+b, & g_{W, i} \geq \theta_{t h}
\end{array},\right.
$$

where $\theta_{t h}=0.2$. We found that the output $g_{W, i}$ of local WTA contained some small peaks that did not correspond to the two peaks in the activity pattern of stimulus receptors (Figure $\mathbf{4 g}$, middle panel). By setting $g_{N, i}=0$ when $g_{W, i}<\theta_{t h}$, we removed these small peaks (Figure 4g, right panel).

The values of $a$ and $b$ in eq. 7 were the same for all $i$ s, and were set so that the heights of the highest two peaks in $\left\{g_{N, i}\right\}_{i}$ were close to the heights of the two peaks in $\left\{x_{i}\right\}_{i}$ (Figure 4g, right panel). To better explain how we fulfill this point, we denote the positions (i.e., indexes) of the highest two peaks in $\left\{g_{W, i}\right\}_{i}$ as $i_{g W 1}$ and $i_{g W 2}$, and the positions of the two peaks in $\left\{x_{i}\right\}_{i}$ as $i_{x 1}$ and $i_{x 2}$. We denote the position of the peak in $\left\{x_{i}\right\}_{i}$ that is closer to $i_{g W 1}$ (or $i_{g W 2}$ ) to be $j_{x 1}$ (or $j_{x 2}$ ), i.e., $j_{x 1}=\arg \min i_{i \in\left\{i_{x 1}, i_{x 2}\right\}}\left(\left|i-i_{g W 1}\right|\right.$ ) (or $\left.j_{x 2}=\arg \min _{i \in\left\{i_{x 1}, i_{x 2}\right\}}\left(\left|i-i_{g W 2}\right|\right)\right)$. We plotted pairs of values $\left(g_{W, i_{g}{ }_{1}}, x_{j_{x 1}}\right)$ and $\left(g_{W, i_{g W 2}}, x_{j_{x 2}}\right)$ for all simulation trials in the same figure, and fit these dots using a linear function. The slop and intercept of this linear function were respectively the parameters $a$ and $b$ in eq. 7 .

\section{Acknowledgments}

Z.B. thanks Prof. Changsong Zhou and Prof. Shuzhi Sam Ge for comments on the manuscript and helpful discussions. Z.B. was supported by the National Natural Science Foundation of China (32000694) and the start-up fund of the Institute for Future of Qingdao University.

\section{References}

[1] K. Friston, “The free-energy principle: a unified brain theory?,” Nat. Rev. Neurosci., vol. 11, pp. 127-138, 2010.

[2] K. Friston, “Does predictive coding have a future?," Nat. Neurosci., vol. 21, pp. 1019-1021, 2018.

[3] R. P. N. Rao and D. H. Ballard, "Predictive coding in the visual cortex: a functional interpretation of some extra-classical receptive-field effects," Nat. Neurosci., vol. 2, pp. 79-87, 1999. 

available under aCC-BY-NC-ND 4.0 International license.

[4] P. Dayan, G. E. Hinton, R. M. Neal, and R. S. Zemel, “The helmholtz machine,” Neural Comput., vol. 7, pp. 889-904, 1995.

[5] B. A. Olshausen and D. J. Field, "Emergence of simple-cell receptive field properties by learning a sparse code for natural images," Nature, vol. 381, pp. 607-609, 1996.

[6] M. Heilbron and M. Chait, "Great expectations: Is there evidence for predictive coding in auditory cortex?," Neuroscience, vol. 389, pp. 54-73, 2018.

[7] N. Dijkstra, L. Ambrogioni, D. Vidaurre, and M. van Gerven, "Neural dynamics of perceptual inference and its reversal during imagery," eLife, vol. 9, p. e53588, 2020.

[8] N. Dijkstra, S. E. Bosch, and M. A. J. van Gerven, "Shared neural mechanisms of visual perception and imagery," Trends Cogn. Sci., vol. 23, pp. 423-434, 2019.

[9] A. M. Albers, P. Kok, I. Toni, H. C. Dijkerman, and F. P. de Lange, "Shared representations for working memory and mental imagery in early visual cortex," Curr. Biol., vol. 23, pp. 1427-1431, 2013.

[10] R. Kanai, A. Chang, Y. Yu, I. M. de Abril, M. Biehl, and N. Guttenberg, "Information generation as a functional basis of consciousness," Neurosci. Conscious., vol. 5, p. niz016, 2019.

[11] W. Wiese, "The science of consciousness does not need another theory, it needs a minimal unifying model," Neurosci. Conscious., vol. 6 , p. niaa013, 2020.

[12] H. Supèr, H. Spekreijse, and V. A. Lamme, "Two distinct modes of sensory processing observed in monkey primary visual cortex (V1)," Nat. Neurosci., vol. 4, pp. 304-310, 2001.

[13] B. van Vugt, B. Dagnino, D. Vartak, H. Safaai, S. Panzeri, S. Dehaene, and P. R. Roelfsema, "The threshold for conscious report: Signal loss and response bias in visual and frontal cortex," Science, vol. 360, pp. 537-542, 2018.

[14] G. A. Mashour, P. Roelfsema, J.-P. Changeux, and S. Dehaene, "Conscious processing and the global neuronal workspace hypothesis," Neuron, vol. 105, pp. 776-798, 2020.

[15] L. Mudrik, N. Faivre, and C. Koch, "Information integration without awareness," Trends Cogn. Sci., vol. 18, pp. 488-496, 2014.

[16] D. Soto and J. Silvanto, "Reappraising the relationship between working memory and conscious awareness," Trends Cogn. Sci., vol. 18, pp. 520-525, 2014.

[17] S. V. Gaal and V. A. F. Lamme, "Unconscious high-level information processing: implication for neurobiological theories of consciousness," Neuroscientist, vol. 18, pp. 287-301, 2012.

[18] J. Pearson, "The human imagination: the cognitive neuroscience of visual mental imagery," Nat. Rev. Neurosci., vol. 20, pp. 624-634, 2019.

[19] B. M. Lake, T. D. Ullman, J. B. Tenenbaum, and S. J. Gershman, "Building machines that learn and think like people," Behav. Brain Sci., vol. 40, p. e253, 2017.

[20] M. G. Mattar and N. D. Daw, "Prioritized memory access explains planning and hippocampal replay,” Nat. Neurosci., vol. 21, pp. 1609$1617,2018$.

[21] R. S. Sutton and A. G. Barto, Reinforcement learning: An introduction. Cambridge: The MIT Press, 2018.

[22] J. L. Breedlove, G. St-Yves, C. A. Olman, and T. Naselaris, "Generative feedback explains distinct brain activity codes for seen and mental images," Curr. Biol., vol. 30, pp. 1-14, 2020.

[23] S. E. Favila, B. A. Kuhl, and J. Winawer, "Perception and memory have distinct spatial tuning properties in human visual cortex," bioRxiv, 2020. 

available under aCC-BY-NC-ND 4.0 International license.

[24] A. Angelucci, M. Bijanzadeh, L. Nurminen, F. Federer, S. Merlin, and P. C. Bressloff, "Circuits and mechanisms for surround modulation in visual cortex," Аnпи. Rev. Neurosci., vol. 40, pp. 425-451, 2017.

[25] A. J. Keller, M. M. Roth, and M. Scanziani, "Feedback generates a second receptive field in neurons of the visual cortex," Nature, vol. 582, pp. 545-549, 2020.

[26] G. C. DeAngelis, R. D. Freeman, and I. Ohzawa, "Length and width tuning of neurons in the cat's primary visual cortex," J. Neurophysiol., vol. 71, pp. 347-374, 1994.

[27] D. Kingma and M. Welling, "Auto-encoding variational bayes," in International Conference on Learning Representations, 2014.

[28] J. D. Murray, A. Bernacchia, N. A. Roy, C. Constantinidis, R. Romo, and X.-J. Wang, "Stable population coding for working memory coexists with heterogeneous neural dynamics in prefrontal cortex," Proc. Natl Acad. Sci. USA, vol. 114, pp. 394-399, 2017.

[29] A. Parthasarathy, C. Tang, R. Herikstad, L. F. Cheong, S.-C. Yen, and C. Libedinsky, "Time-invariant working memory representations in the presence of code-morphing in the lateral prefrontal cortex," Nat. Commun., vol. 10, p. 4995, 2019.

[30] S. Ganguli and H. Sompolinsky, "Compressed sensing, sparsity, and dimensionality in neuronal information processing and data analysis," Аnпи. Rev. Neurosci., vol. 14, pp. 485-508, 2012.

[31] M. Rani, S. B. Dhok, and R. B. Deshmukh, “A systematic review of compressive sensing: Concepts, implementations and applications," IEEE Access, vol. 6, pp. 4875-4894, 2018.

[32] E. D. de Jong, "Incremental sequence learning," arXiv:1611.03068, 2016.

[33] W. Li, V. Piëch, and C. D. Gilbert, "Contour saliency in primary visual cortex,” Neuron, vol. 50, pp. 951-962, 2006.

[34] K. Anton-Erxleben and M. Carrasco, "Attentional enhancement of spatial resolution: linking behavioural and neurophysiological evidence," Nat. Rev. Neurosci., vol. 14, pp. 188-200, 2013.

[35] N. D. Pisapia, F. Bacci, D. Parrott, and D. Melcher, "Brain networks for visual creativity: a functional connectivity study of planning a visual artwork," Sci. Rep., vol. 6, p. 39185, 2016.

[36] T. Salimans, I. Goodfellow, W. Zaremba, V. Cheung, A. Radford, and X. Chen, "Improved techniques for training GANs," in Proceedings of the 30th International Conference on Neural Information Processing Systems, pp. 2234-2242, 2016.

[37] K. Rajan, L. Abbott, and H. Sompolinsky, "Inferring stimulus selectivity from the spatial structure of neural network dynamics," in Advances in Neural Information Processing Systems, vol. 23, 2010.

[38] K. Perlin, “Noise hardware,” in Real-Time Shading SIGGRAPH Course Notes (M. Olano, ed.), 2001.

[39] A. Safia and D.-C. He, "New brodatz-based image databases for grayscale color and multiband texture analysis," ISRN Machine Vision, vol. 21, p. 876386, 2013.

[40] J. Rabin, G. Peyr, J. Delon, and M. Bernot, "Wasserstein barycenter and its application to texture mixing," in Scale Space and Variational Methods in Computer Vision (SSVM), pp. 435-446, 2011.

[41] T. Karras, T. Aila, S. Laine, and J. Lehtinen, "Progressive growing of GANs for improved quality, stability, and variation," in The International Conference on Learning Representations, 2018.

[42] I. Higgins, L. Matthey, A. Pal, C. Burgess, X. Glorot, M. Botvinick, S. Mohamed, and A. Lerchner, "beta-VAE: Learning basic visual concepts with a constrained variational framework," in The International Conference on Learning Representations, 2017.

[43] E. H. Nieh, M. Schottdorf, N. W. Freeman, R. J. Low, S. Lewallen, S. A. Koay, L. Pinto, J. L. Gauthier, C. D. Brody, and D. W. Tank, "Geometry of abstract learned knowledge in the hippocampus," Nature, vol. 595, pp. 80-84, 2021. 

available under aCC-BY-NC-ND 4.0 International license.

[44] Y. Fang, X. Wang, S. Zhong, L. Song, Z. Han, G. Gong, and Y. Bi, "Semantic representation in the white matter pathway," PLoS Biol., vol. 16, p. e2003993, 2018.

[45] A. O. Constantinescu, J. X. O’Reilly, and T. E. J. Behrens, "Organizing conceptual knowledge in humans with a gridlike code," Science, vol. 352, pp. 1464-1468, 2016.

[46] L. Itti and C. Koch, “Computational modelling of visual attention,” Nat. Rev. Neurosci., vol. 2, pp. 194-203, 2001.

[47] C. D. Gilbert and W. Li, “Top-down influences on visual processing,” Nat. Rev. Neurosci., vol. 14, pp. 350-363, 2013.

[48] A. Radford, L. Metz, and S. Chintala, "Unsupervised representation learning with deep convolutional generative adversarial networks," in The International Conference on Learning Representations, 2016.

[49] A. B. L. Larsen, S. K. S $\phi$ nderby, H. Larochelle, and O. Winther, “Autoencoding beyond pixels using a learned similarity metric,” in Proceedings of Machine Learning Research, 2016.

[50] D. P. Kingma, D. J. Rezendey, S. Mohamedy, and M. Welling, "Semi-supervised learning with deep generative models," in Conference on Neural Information Processing Systems, 2014.

[51] M. E. Abbasnejad, A. Dick, and A. van den Hengel, "Infinite variational autoencoder for semi-supervised learning," in Conference on Computer Vision and Pattern Recognition, 2017.

[52] I. Goodfellow, Y. Bengio, and A. Courville, Deep learning. Cambridge: The MIT Press, 2016.

[53] A. Odena, C. Olah, and J. Shlens, "Conditional image synthesis with auxiliary classifier GANs," in International Conference on Machine Learning, 2017.

[54] M. Arjovsky and L. Bottou, “Towards principled methods for training generative adversarial networks," in The International Conference on Learning Representations, 2017.

[55] A. C. Nobre and F. van Ede, “Anticipated moments: temporal structure in attention,” Nat. Rev. Neurosci., vol. 19, pp. 34-48, 2018.

[56] J. M. Wolfe and T. S. Horowitz, "Five factors that guide attention in visual search," Nat. Hum. Behav., vol. 1, p. 0058, 2017.

[57] L. H. Arnal, V. Wyart, and A.-L. Giraud, "Transitions in neural oscillations reflect prediction errors generated in audiovisual speech," Nat. Neurosci., vol. 14, pp. 797-801, 2011.

[58] A. M. Bastos, J. Vezoli, C. A. Bosman, J.-M. Schoffelen, R. Oostenveld, J. R. Dowdall, P. D. Weerd, H. Kennedy, and P. Fries, "Visual areas exert feedforward and feedback influences through distinct frequency channels," Neuron, vol. 85, pp. 390-401, 2015.

[59] C. Stringer, M. Pachitariu, N. Steinmetz, M. Carandini, and K. D. Harris, "High-dimensional geometry of population responses in visual cortex," Nature, vol. 571, pp. 361-365, 2019.

[60] D. P. Kingma and J. Ba, "Adam: A method for stochastic optimization," in The International Conference on Learning Representations, 2015. 